\title{
Research Progress on the Photocatalytic Conversion of Methane and Methanol
}

\author{
ZHANG Shuyi ${ }^{1,2}$, BAO Jingxian ${ }^{1,3}$, WU Bo ${ }^{1,2}$, ZHONG Liangshu ${ }^{1,4, *}$, SUN Yuhan 1,4,* \\ ${ }^{1}$ CAS Key Laboratory of Low-Carbon Conversion Science and Engineering, Shanghai Advanced Research Institute, \\ Chinese Academy of Sciences, Shanghai 201203, P. R. China. \\ ${ }^{2}$ University of the Chinese Academy of Sciences, Beijing 100049, P. R. China. \\ ${ }^{3}$ College of Sciences, Shanghai University, Shanghai 200444, P. R. China. \\ ${ }^{4}$ School of Physical Science and Technology, ShanghaiTech University, Shanghai 201203, P. R. China.
}

\begin{abstract}
With the increasing energy demands and the limited petroleum reserves, it is highly desirable to produce fuels and chemicals from non-petroleum feedstocks, such as coal, natural gas and biomass. Catalytic conversion of $\mathrm{C}_{1}$ resources $\left(\mathrm{CO}, \mathrm{CO}_{2}\right.$, $\mathrm{CH}_{3} \mathrm{OH}, \mathrm{CH}_{4}$, etc.) affords various products and attracts increasing attention from both academia and industries. Methane and methanol are important $C_{1}$ feedstocks in the production of fuels and chemicals. In order to obtain high selectivity for the target product, it is necessary to control the activation of $\mathrm{C}-\mathrm{H}$ bonds in methane and methanol. However, this remains a great challenge. Although the traditional thermal catalytic conversion of methane and methanol has been developed over decades, there are still some disadvantages associated with the catalytic process, such as harsh reaction conditions, high energy consumption, and low selectivity. Photocatalysis,

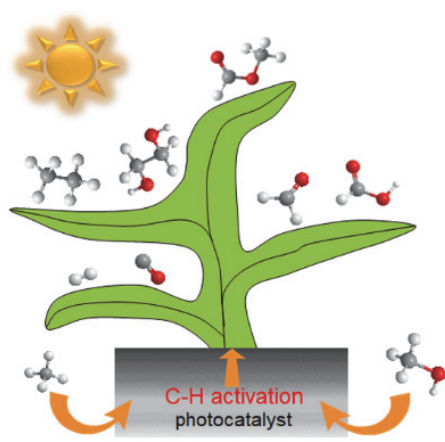
which is driven by photoenergy, can compensate for the Gibbs free energy. In the photocatalytic reactions, semiconductor photocatalysts absorb photons and generate electrons and holes in their conduction and valence bands, respectively, to accelerate the reaction rate. The position of the conduction band determines the oxidation capacity, and the bandgap determines the light absorption property. Normally, the oxidation capacity of photocatalysts is regulated by choosing semiconductors with a suitable bandgap or anions/cations doping. Fabrication of heterojunction and loading metalsare recognized as effective methods to promote the separation of electron-hole pairs and improve the photocatalytic efficiency. In contrast to thermal catalysis, photocatalysis can be carried out under mild reaction conditions with low energy consumption. Recently, photocatalysis has been considered an attractive route for the efficient conversion of methane and methanol to fuels and chemicals. Partial oxidation of methane, which is necessary to avoid the formation of byproducts, can be achieved by adjusting the wavelength and intensity of the light and the oxidation capacity of the photocatalysts. In addition, light-induced plasmon resonance improves the efficiency of methane conversion by forming an intrinsic highenergy magnetic field that can polarize methane. In methanol conversion, the $\mathrm{C}-\mathrm{H}$ bond can be selectively activated, instead of the $\mathrm{O}-\mathrm{H}$ bond, by light irradiation. Therefore, $\mathrm{C}-\mathrm{C}$ coupling can be realized for the production of various valueadded chemicals from methanol. This review summarizes the recent advances in the photocatalytic conversion of methane and methanol including the reactions of reforming, oxidation, and coupling. Perspectives and challenges for further research on the photocatalytic conversion of methane and methanol are also discussed.
\end{abstract}

Key Words: Activation of $\mathrm{C}-\mathrm{H}$ bond; $\mathrm{C}_{1}$ chemistry; Methane; Methanol; Photocatalysis

Received: October 8, 2018; Revised: November 21, 2018; Accepted: November 21, 2018; Published online: November 26, 2018.

${ }^{\star}$ Corresponding authors. Emails: zhongls@sari.ac.cn (Z.L.); sunyh@sari.ac.cn (S.Y.). Tel.: + 86-21-20608002 (Z.L.); +86-21-20325009 (S.Y.).

This work was supported by the National Key R\&D Program of China (2017YFB0602202, 2018YFB0604700), National Natural Science Foundation of China (21573271, 91545112, 21703278), Key Research Program of Frontier Sciences, CAS (QYZDB-SSW-SLH035), the "Transformational Technologies for Clean Energy and Demonstration" and Strategic Priority Research Program of the Chinese Academy of Sciences (XDA21020600).

国家重点研发计划重点专项(2017YFB0602202, 2018YFB0604700), 国家自然科学基金(21573271, 91545112, 21703278), 中国科学院前沿科学重点研究 计划(QYZDB-SSW-SLH035), 中国科学院洁净能源先导科技专项(XDA21020600)资助

(C) Editorial office of Acta Physico-Chimica Sinica 


\section{甲烷/甲醇光催化转化研究进展}

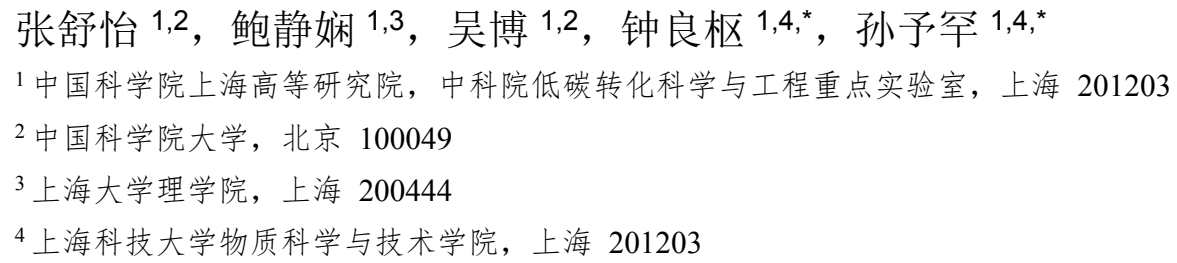

摘要: 在能源需求不断上涨及石油供应日益紧张的背景下, 开展对煤、天然气或生物质等非油基资源 $\left(\mathrm{CO} 、 \mathrm{CO}_{2} 、 \mathrm{CH}_{3} \mathrm{OH}\right.$ 、 $\mathrm{CH}_{4}$ 等)的高效利用显得尤为重要。 $\mathrm{C}_{1}$ 小分子 $\left(\mathrm{CO} 、 \mathrm{CO}_{2} 、 \mathrm{CH}_{3} \mathrm{OH} 、 \mathrm{CH}_{4}\right.$ 等)经催化转化可得到燃料及多种化学品, 一直受 到学术界及工业界的广泛关注。甲烷/甲醇作为重要的 $\mathrm{C}_{1}$ 平台分子, 其催化转化在 $\mathrm{C}_{1}$ 化学中占据重要地位。为了提高目标 产物的选择性, 需要有效地控制甲烷/甲醇中 $\mathrm{C}-\mathrm{H}$ 键的活化。传统热催化作为甲烷/甲醇最常见的转化方法发展已久, 但 仍然面临着反应条件苛刻、能耗大、产率和选择性低等问题。光催化反应通过引入光能弥补反应中吉布斯自由能的上升, 同时具有反应条件温和、操作简单、能耗低等特点, 从而为甲烷/甲醇转化提供了新的途径。通过调节光的波长、强度以 及催化剂的氧化能力可以实现甲烷/甲醇的选择性转化, 减少副产物的生成。此外, 光催化能够选择性活化甲醇的 $\mathrm{C}-\mathrm{H}$ 键而非 $\mathrm{O}-\mathrm{H}$ 键, 从而实现甲醇的 $\mathrm{C}-\mathrm{C}$ 偶联反应。本文主要围绕甲烷/甲醇的重整、氧化和偶联反应, 总结近年来的光催 化转化进展, 并对进一步提高光催化性能做了展望。

关键词: $\mathrm{C}-\mathrm{H}$ 键活化; $\mathrm{C}_{1}$ 化学; 甲烷; 甲醇; 光催化 中图分类号: 0644

\section{1 引言}

$\mathrm{C}-\mathrm{H}$ 键是有机化合物中最常见的价键结构, 通 过 $\mathrm{C}-\mathrm{H}$ 键高效活化与定向转化 ${ }^{1-3}$, 可进行包括嗍 化 ${ }^{4,5}$ 、内胺化 ${ }^{6}$ 和澳化 ${ }^{7}$ 等一系列反应, 进而得到各 类高附加值产物。由于 $\mathrm{C}-\mathrm{H}$ 键的惰性和产物的多 样性, 实现 $\mathrm{C}-\mathrm{H}$ 键高效活化与定向转化一直是化 学家的梦想。为了解决低活性及低选择性两大难 题, 通常采用三种方法: 一是底物控制法, 依靠底 物本身不同 $\mathrm{C}-\mathrm{H}$ 键键能之间的差异而实现高选 择性, 但此方法受到底物种类限制; 二是导向控制 法, 在目标分子上连接一个导向基, 随后此导向基 引导催化剂将目标键切断, 该方法目前较为成熟, 但需要涉及导向基的功能化和去除两个步骤 ${ }^{8}$; 三 是催化剂控制法, 通过设计具有独特结构的催化
剂摆脱对底物的依赖, 实现对各类 $\mathrm{C}-\mathrm{H}$ 键的识别 选择 ${ }^{9-11}$, 目前此方法越来越受到人们关注。

与热催化相比, 光催化具有反应条件温和、操 作简单、能耗低等优点。光催化活化 $\mathrm{C}-\mathrm{H}$ 键目前 正处于快速发展期, 常采用的均相光催化反应通 常是由激发态的光敏剂激发分子产生自由基, 再 进行单电子转移和质子转移两种催化循环 ${ }^{12-19}$ 。此 外, 半导体非均相光催化剂也受到越来越多的关 注 ${ }^{20-23}$ 。 $\mathrm{C}_{1}$ 催化涉及 $\mathrm{C}_{1}$ 分子 $\left(\mathrm{CO} 、 \mathrm{CO}_{2} 、 \mathrm{CH}_{3} \mathrm{OH} 、 \mathrm{CH}_{4}\right.$ 等)转化, 是煤、天然气或生物质等非油基资源转 化利用的一条重要途径。在原油供应日趋紧张和 国家需求持续高涨的大背景下, 开展 $\mathrm{C}_{1}$ 定向催化 转化制高品质液体燃料和高附加值化学品研究工 作对满足国家重大需求、优化利用化石资源有着

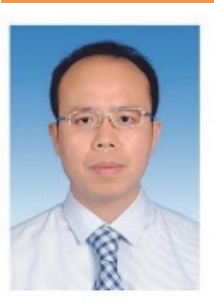

钟良枢, 2003年本科毕业于北京化 工大学, 获应用化学学士学位; 2008 年6月毕业于中国科学院化学研究 所, 获物理化学博士学位; 2008年7 月至2010年6月在德国慕尼黑工业 大学化学系进行博士后研究。现任 中国科学院上海高等研究院研究员。主要从事 $\mathrm{C}_{1}$ 分 子高选择性转化制清洁燃料与高附加值化学品相关 的催化研究。

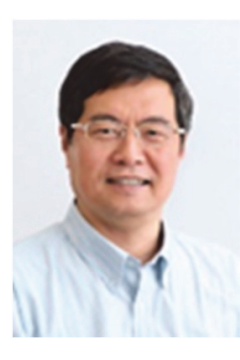

孙予罕, 1983年毕业于郑州大学化 学系, 获得学士学位; 1983年至 1989年在中国科学院山西煤炭化 学研究所获博士学位。现任中国科 学院上海高等研究院低碳转化科 学与工程重点实验室主任。主要从 事含碳资源与 $\mathrm{CO}_{2}$ 转化利用中催 化和工程研究, 以及相关纳米材料及其在绿色化学 中的应用研究, 包括近期的清洁能源战略与解决方 案研究。 
重要意义。甲烷、甲醇作为重要的 $\mathrm{C}_{1}$ 分子, 其催化 转化皆涉及 $\mathrm{C}-\mathrm{H}$ 键活化。为了实现更高的产物选 择性, 需要很好地控制 $\mathrm{C}-\mathrm{H}$ 键活化及 $\mathrm{C}-\mathrm{C}$ 键形 成, 而光催化是一条极具潜力的解决途径。本综述 对近年来甲烷/甲醇光催化转化相关进展进行简要 评述。

\section{2 甲烷光催化转化}

甲烷是天然气、页岩气、沼气和可燃冰等含碳 资源的主要组分, 相比煤炭及石油, 具有更高氢碳 比, 可视为一种清洁能源。除了直接作为燃料, 将 甲烷催化转化为高附加值产物有利于实现甲烷高 效利用, 具有重要的学术意义及工业应用价值。

甲烷是高度对称分子, 是最稳定的碳氢化合 物, 其 $\mathrm{C}-\mathrm{H}$ 键断裂能量高达 $434 \mathrm{~kJ} \cdot \mathrm{mol}^{-1}$, 活化能 较高, 因此 $\mathrm{C}-\mathrm{H}$ 键活化是甲烷转化的速率控制步
骤。热催化作为传统甲烷转化方法近年来被广泛 研究, 但是一般需要苛刻的反应条件, 因此寻找其 它高效的甲烷活化方法成为当前研究热点 ${ }^{24-27}$ 。光 催化反应由光能激发产生高能电子和空穴, 参与 到甲烷 $\mathrm{C}-\mathrm{H}$ 键活化和自由基形成, 进而补偿反应 吉布斯自由能上升。此外, 光催化反应条件通常在 较为温和条件下进行, 这为低温转化甲烷提供新 的途径。本节主要介绍基于光催化的甲烷重整、部 分氧化及偶联反应 ${ }^{28-39}$ (表1)。

\section{1 甲烷光催化重整}

重整反应是甲烷直接制合成气 $\left(\mathrm{CO}+\mathrm{H}_{2}\right)$ 最常 用的方法。甲烷重整分为甲烷干重整 (dry reforming of methane, DRM)和甲烷湿重整(steam reforming of methane, SRM), 干重整是甲烷与二 氧化碳反应, 而湿重整是甲烷与水蒸气反应 ${ }^{40-42}$ 。 相比湿重整, 干重整利用二氧化碳作为原料制备

\section{表1 各类甲烷光催化反应催化性能的汇总}

Table 1 Catalytic performances for methane conversion over different photocatalysts.

\begin{tabular}{|c|c|c|c|c|c|c|c|c|c|c|}
\hline \multirow{2}{*}{ Entry } & \multirow{2}{*}{ Catalyst } & \multirow{2}{*}{ Reaction } & \multicolumn{4}{|c|}{ Reaction conditions } & \multicolumn{3}{|c|}{ Catalytic performance } & \multirow{2}{*}{ Ref. } \\
\hline & & & $T /{ }^{\circ} \mathrm{C}$ & $P / \mathrm{MPa}$ & Light source & $I /\left(\mathrm{mW} \cdot \mathrm{cm}^{-2}\right)$ & Reaction rate & Conv. $\%$ & Sel./\% & \\
\hline \multirow[t]{2}{*}{1} & $\mathrm{Rh}-\mathrm{Au} / \mathrm{SBA}-15$ & methane to & 500 & 0.1 & LA-251Xe-lamp & 280 & $1650-1750 \mu \mathrm{mol}_{\mathrm{CH}_{4}} \cdot \mathrm{g}^{-1} \cdot \mathrm{s}^{-1}$ & NA & $\sim 100$ & 28 \\
\hline & & syngas & & & (HA30 filter) & & $2150-2200 \mu \mathrm{mol}_{\mathrm{CO}_{2}} \cdot \mathrm{g}^{-1} \cdot \mathrm{s}^{-1}$ & & & \\
\hline \multirow[t]{2}{*}{2} & $\mathrm{Pt} /$ black- $-\mathrm{TiO}_{2}$ & methane to & 650 & 0.1 & sunlight & 100 & $129 \mathrm{mmol}_{\mathrm{H}_{2}} \cdot \mathrm{g}^{-1} \cdot \mathrm{h}^{-1}$ & NA & NA & 29 \\
\hline & & syngas & & & & & $103 \mathrm{mmol}_{\mathrm{CO}} \cdot \mathrm{g}^{-1} \cdot \mathrm{h}^{-1}$ & & & \\
\hline \multirow[t]{2}{*}{3} & $\mathrm{Ni} / \mathrm{SiO}_{2}-\mathrm{Im}$ & methane to & 550 & 0.1 & LA-251Xe-lamp & 1070 & $1270 \mu \mathrm{mol}_{\mathrm{H}_{2}} \cdot \mathrm{g}_{\mathrm{Ni}}^{-1} \cdot \min ^{-1}$ & NA & $\sim 100$ & 30 \\
\hline & & syngas & & & (HA30 filter) & & $1270 \mu \mathrm{mol}_{\mathrm{H}_{2}} \cdot \mathrm{g}_{\mathrm{Ni}}^{-1} \cdot \mathrm{min}^{-1}$ & & & \\
\hline \multirow[t]{2}{*}{4} & $\mathrm{Pt}-\mathrm{Si}-\mathrm{CeO}_{2}$ & methane to & 600 & 0.1 & solar simulator & 3000 & $90 \mathrm{mmol}_{\mathrm{H}_{2}} \cdot \mathrm{g}^{-1} \cdot \mathrm{h}^{-1}$ & 25 & NA & 31 \\
\hline & & syngas & & & & & $154 \mathrm{mmol}_{\mathrm{CO}} \cdot \mathrm{g}^{-1} \cdot \mathrm{h}^{-1}$ & & & \\
\hline \multirow[t]{2}{*}{5} & $\mathrm{Ni} / \mathrm{Al}_{2} \mathrm{O}_{3}$ & methane to & 550 & 0.1 & LA-251Xe-lamp & 1070 & $132 \mu \mathrm{mol}_{\mathrm{H}} \cdot \mathrm{g}^{-1} \cdot \mathrm{h}^{-1}$ & NA & $\sim 100$ & 32 \\
\hline & & syngas & & & (HA30 filter) & & $130 \mu \mathrm{mol}_{\mathrm{CO}} \cdot \mathrm{g}^{-1} \cdot \mathrm{h}^{-1}$ & & & \\
\hline \multirow[t]{2}{*}{6} & $\mathrm{Bi}-\mathrm{V} / \mathrm{BETA}$ & methane to & 70 & 0.1 & Hg lamp & NA & $10.7 \mu \mathrm{mol}_{\mathrm{CH}_{3} \mathrm{OH}} \cdot \mathrm{g}^{-1} \cdot \mathrm{h}^{-1}$ & NA & NA & 33 \\
\hline & & methanol & & & & & & & & \\
\hline \multirow[t]{2}{*}{7} & Beta & methane to & RT & 0.5 & deep-UV & NA & NA & 6.3 & 5.4 & 34 \\
\hline & zeolite & methanol & & & $(185 \mathrm{~nm})$ & & & & & \\
\hline \multirow[t]{2}{*}{8} & V-MCM-41 & methane to & 300 & 0.1 & Hg lamp UV & NA & $9.6 \mu \mathrm{mol}_{\mathrm{CH}_{3} \mathrm{OH}} \cdot \mathrm{g}^{-1} \cdot \mathrm{h}^{-1}$ & 7.1 & 88 & 35 \\
\hline & & methanol & & & $(>270 \mathrm{~nm})$ & & & & & \\
\hline \multirow[t]{2}{*}{9} & $n$-GaN NWs & methane to & 5 & 0.1 & Xe lamp UV & 7.5 & $11.39 \times 10 \mu \mathrm{mol}_{\mathrm{CH}_{4}} \cdot \mathrm{g}^{-1} \cdot \mathrm{h}^{-1}$ & NA & 96 & 36 \\
\hline & & benzene & & & $(290-380 \mathrm{~nm})$ & & $1.77 \times 10^{-2} \mu \mathrm{mol}_{\mathrm{C}_{6} \mathrm{H}_{6}} \cdot \mathrm{g}^{-1} \cdot \mathrm{h}^{-1}$ & & & \\
\hline \multirow[t]{2}{*}{10} & $\mathrm{Au} / \mathrm{ZnO}$ & methane to & RT & 0.1 & Xe lamp & 600 & $11.25 \mu \mathrm{mol}_{\mathrm{C}_{2} \mathrm{H}_{6}} \cdot \mathrm{g}^{-1} \cdot \mathrm{h}^{-1}$ & NA & NA & 37 \\
\hline & & ethane & & & $(300-800 \mathrm{~nm})$ & & $10 \mu \mathrm{mol}_{\mathrm{H}_{2}} \cdot \mathrm{g}^{-1} \cdot \mathrm{h}^{-1}$ & & & \\
\hline \multirow[t]{2}{*}{11} & $\left(\mathrm{Zn}^{+}, \mathrm{Zn}^{2+}\right) / \mathrm{ZSM}-5$ & methane to & RT & 0.1 & Hg lamp & 100 & $9.8 \mathrm{mmol}_{\mathrm{CH}_{4}} \cdot \mathrm{g}^{-1} \cdot \mathrm{h}^{-1}$ & 24 & 99 & 38 \\
\hline & & ethane & & & $(<270 \mathrm{~nm})$ & & $4.9 \mathrm{mmol}_{\mathrm{H}_{2}} \cdot \mathrm{g}^{-1} \cdot \mathrm{h}^{-1}$ & & & \\
\hline \multirow[t]{2}{*}{12} & $\mathrm{Ga}^{3+}-\mathrm{EST}-10$ & methane to & RT & 0.1 & Hg lamp & 100 & $29.8 \mu \mathrm{mol}_{\mathrm{CH}_{4}} \cdot \mathrm{g}^{-1} \cdot \mathrm{h}^{-1}$ & 14.9 & NA & 39 \\
\hline & & ethane & & & $(<370 \mathrm{~nm})$ & & & & & \\
\hline
\end{tabular}



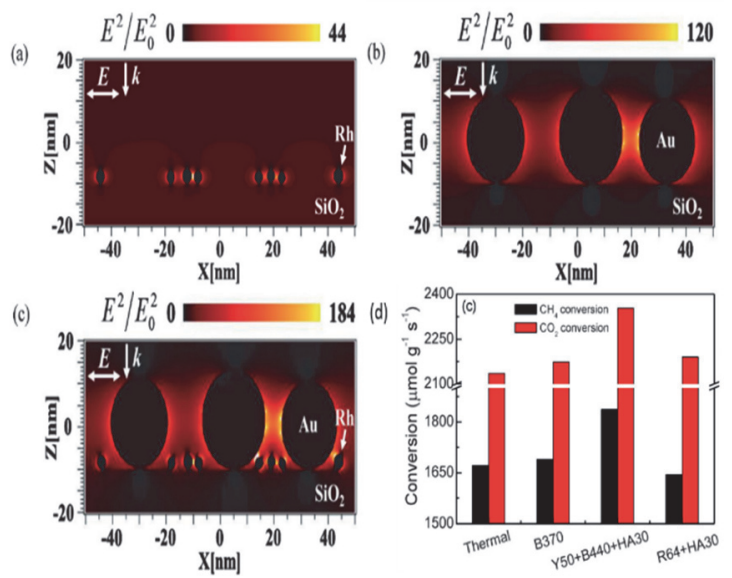

图1 (a) Rh/SBA-15, (b) Au/SBA-15和(c) Rh-Au/SBA-15 有限差分时域方法下的电磁场分部和增强刺激交联

区域视图; (d)不同波长下 $\mathrm{CH}_{4}$ 和 $\mathrm{CO}_{2}$ 转化速率

Fig. 1 Cross-sectional views of the electromagnetic field distribution and enhancement simulated with FDTD (finite-difference time-domain) method of (a) Rh/SBA-15,

(b) Au/SBA-15, (c) Rh-Au/SBA-15; (d) $\mathrm{CH}_{4}$ and $\mathrm{CO}_{2}$ conversion rate without or with light irradiation of different wavelength ranges.

Reproduced with permission from reference ${ }^{28}$.

合成气，可视为一条有效的二氧化碳减排途径。但 是, 干重整反应需要更高反应温度 $\left(800^{\circ} \mathrm{C}\right.$ 以上 $)$, 无 疑会增加反应能耗并对催化剂的热稳定性提出更
高要求。

光能的引入能够显著降低DRM的反应启动温 度。贵金属如 $\mathrm{Rh} 、 \mathrm{Pt}$ 等具有较好的DRM反应活性。 $\mathrm{Ye}$ 等 $^{28}$ 选用 $\mathrm{Rh}$ 为主要活性组分, $\mathrm{Au}$ 为助催化剂, 制 备 Rh-Au/SBA-15 双金属催化剂并用于光催化 DRM反应。有限差分时域(FDTD)实验表明在530 $\mathrm{nm}$ 下, $\mathrm{Rh} / \mathrm{SBA}-15$ 催化剂上电磁场强度 $\left(\left|E^{2}\right|\right)$ 非 常弱, $E^{2} / E_{0}^{2}$ 仅为 44 , 而 $\mathrm{Au} / \mathrm{SBA}-15$ 的电磁场强度较 强, $E^{2} / E_{0}^{2}$ 高达 120 ; 在 Rh/SBA-15催化剂上加入 $\mathrm{Au}$ 之后, 由于 $\mathrm{Rh}$ 与 $\mathrm{Au}$ 纳米粒子之间的耦合作用, $E^{2} / E_{0}^{2}$ 提升至180 (图1a-c)。因此, Rh-Au/SBA-15具有更 强的极化甲烷的能力。如图 $1 \mathrm{~d}$ 所示, 光照下反应活 性较无光条件均有一定程度提高, 其中以Y50+ $\mathrm{B} 440+\mathrm{HA} 30$ (光波长范围为 $490-550 \mathrm{~nm}$ ) 为滤光 片时, 具有最佳性能。在 $500{ }^{\circ} \mathrm{C}$ 及光强度为 0.28 $\mathrm{W} \cdot \mathrm{cm}^{-2}$ 时, $\mathrm{Rh}-\mathrm{Au} / \mathrm{SBA}-15$ 的甲烷转化速率达 1750 $\mu \mathrm{mol} \cdot \mathrm{g}^{-1} \cdot \mathrm{s}^{-1}, \mathrm{CO}_{2}$ 转化速率达 $2200 \mu \mathrm{mol} \cdot \mathrm{g}^{-1} \cdot \mathrm{s}^{-1}$ 。

$\mathrm{Pt}$ 作为贵金属在反应中具有较好的电子传输 性能和 $\mathrm{C}-\mathrm{H}$ 键断裂能力。 $\mathrm{Hu}$ 等 ${ }^{29}$ 合成 $\mathrm{Pt} / \mathrm{TiO}_{2}$ 催化 剂, 研究其DRM反应性能。如图 $2 a, b$ 所示, 与无 光与可见光条件下相比, $\mathrm{AM} 1.5 \mathrm{G}$ 效果最佳, 可将 反应启动温度降至 $350{ }^{\circ} \mathrm{C}$, 且 $\mathrm{H}_{2}$ 和 $\mathrm{CO}$ 生成速率显 著提升。当温度升高至 $650{ }^{\circ} \mathrm{C}$ 时, $\mathrm{H}_{2}$ 和 $\mathrm{CO}$ 生成速 率达到 129 和 $370 \mathrm{mmol} \cdot \mathrm{h}^{-1} \cdot \mathrm{g}^{-1}$, 且光量子产率 $(\mathrm{QE})$ 最高。从图2c中可以看到, 在 $650{ }^{\circ} \mathrm{C}$ 之前 $\mathrm{QE}$ 随着
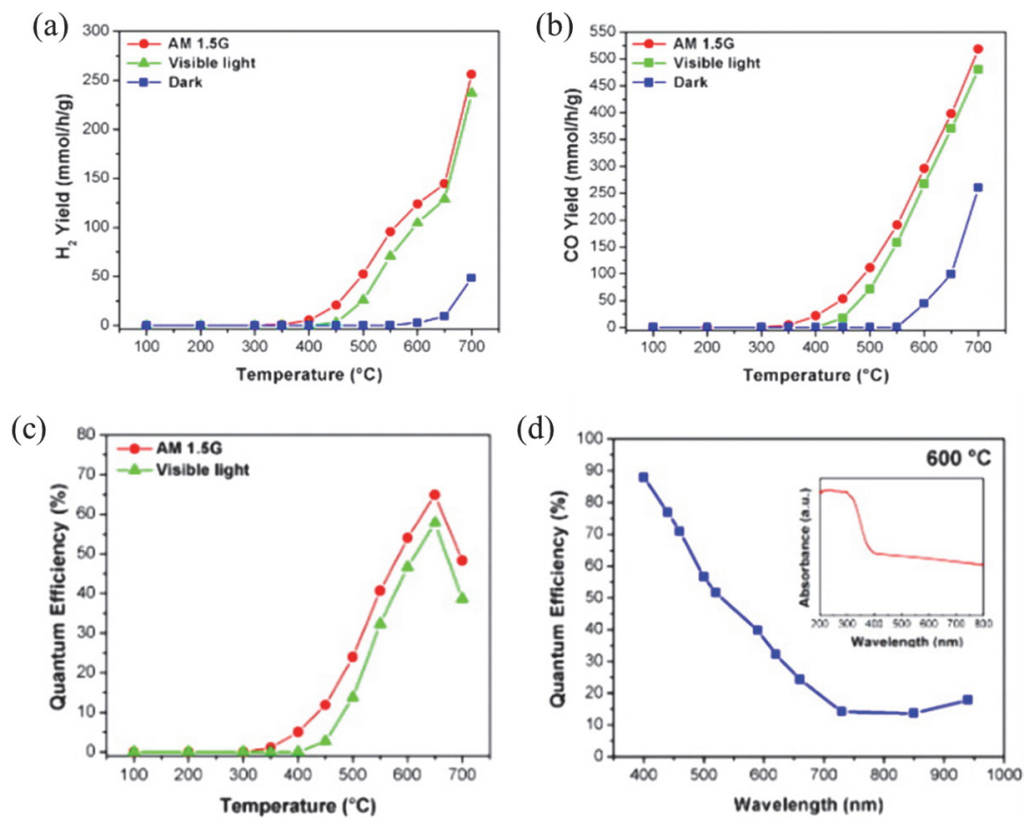

图2 在DRM反应中, 黑色 $\mathrm{TiO}_{2}$ 催化剂 (a) $\mathrm{H}_{2}$ 和(b) $\mathrm{CO}$ 的收率随时间变化图, (c)量子产率随反应温度变化图和

(d) 量子产率随波长变化趋势图

Fig. 2 (a) $\mathrm{H}_{2}$ and (b) CO over Pt/black $\mathrm{TiO}_{2}$ catalyst for DRM reaction, (c) Quantum efficiency (QE) versus temperature (T) and (d) QE versus wavelength ( $(\lambda)$. 
温度上升而提高, 这是因为 $\mathrm{CO}_{2} / \mathrm{CO}$ 和 $\mathrm{CO} / \mathrm{CH}_{4}$ 两个 半反应的过电势随着温度升高而增加。而在 $650^{\circ} \mathrm{C}$ 以后 $\mathrm{QE}$ 有所下降, 这是因为热催化活性急剧上升, 热催化起主要作用, 光催化作用贡献降低。另外, 波长也是影响 $\mathrm{QE}$ 的重要因素。如图2d所示, 低波 长的光源具有更高 $\mathrm{QE}$, 这表明低波长的光子能够 激发电子跃迁到更高能级, 增加过电势以活化甲 烷, 从而提高 $\mathrm{CO}$ 和 $\mathrm{H}_{2}$ 生成速率。

$\mathrm{Rh} 、 \mathrm{Pt}$ 等贵金属催化剂虽然具有较好的 DRM 催化性能, 但是由于其储量较少且价格昂贵, 催化 剂成本高。因此发展高效、价格低廉的非贵金属催 化剂意义重大。非贵金属 $\mathrm{Ni}$ 基催化剂具有较高 DRM反应活性的同时也具有一定的光诱导等离子 体共振效应 ${ }^{43-45}$ 。

$\mathrm{Ye}$ 等 ${ }^{30}$ 采用碳纳米管为模板制备 $\mathrm{SiO}_{2}$ 负载 $\mathrm{Ni}$ 的核壳 $\left(\mathrm{Ni} @ \mathrm{SiO}_{2}\right.$-core) 和蛋壳 $\left(\mathrm{Ni} @ \mathrm{SiO}_{2}\right.$-yolk) 结构 催化剂, 同时采用等体积浸渍法制备 $\mathrm{Ni} / \mathrm{SiO}_{2}-\mathrm{Im}$ 催 化剂。研究结果表明, 光照后三种催化剂反应性能 都显著提高(图3a)。其中, $\mathrm{Ni} / \mathrm{SiO}_{2}$-Im在反应前 $2 \mathrm{~h}$ 内活性最高, 这是由于 $\mathrm{Ni}$ 在 $\mathrm{SiO}_{2}$ 上高度分散, 具有
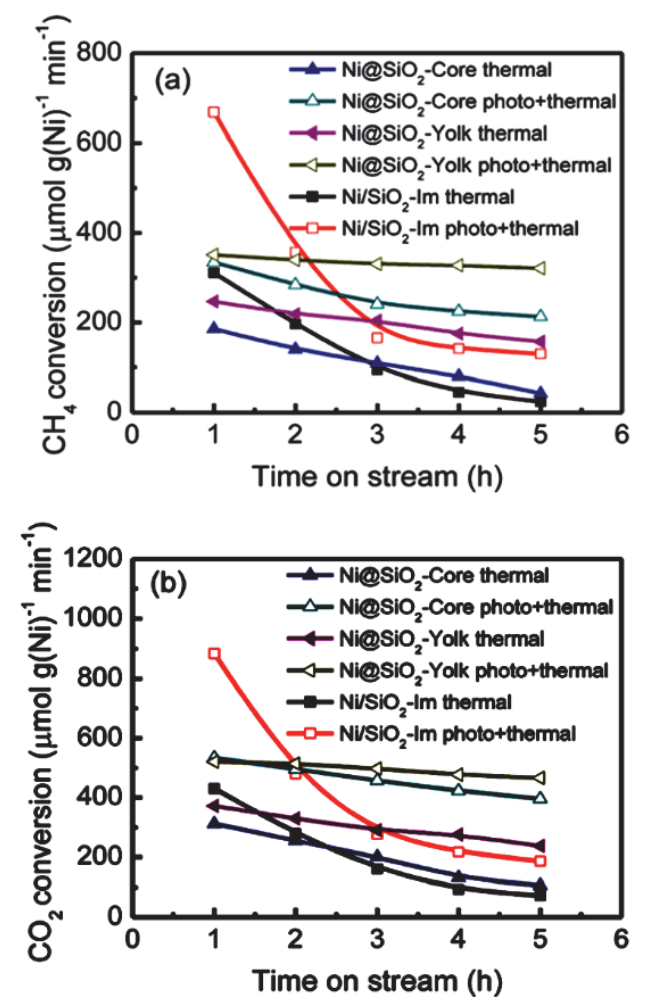

图3 (a)不同滤光片的光谱图,

(b) $\mathrm{Ni} / \mathrm{SiO}_{2}-\mathrm{Im}$ 催化剂在不同波长光照下的反应活性

Fig. 3 (a) Spectra of the light source obtained with different filter sets, (b) reaction activities of

$\mathrm{Ni} / \mathrm{SiO}_{2}-\mathrm{Im}$ catalyst with various light irradiation sources.

Reproduced with permission from reference ${ }^{30}$.
较小的尺寸从而暴露更多活性位, 提高了DRM反 应活性。但是随着反应时间延长, 其活性下降较 快, 反应稳定性远不及 $\mathrm{Ni} @ \mathrm{SiO}_{2}$-yolk和 $\mathrm{Ni} @ \mathrm{SiO}_{2}-$ core (图3b)。FDTD显示在 $430 \mathrm{~nm}$ 条件下, 磁场强

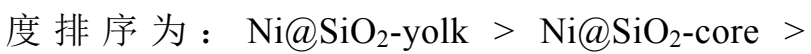
$\mathrm{Ni} / \mathrm{SiO}_{2}-\mathrm{Im}$, 与甲烷光热催化反应活性顺序 $\left(\mathrm{Ni} / \mathrm{SiO}_{2}-\mathrm{Im}>\mathrm{Ni} @ \mathrm{SiO}_{2}\right.$-core $>\mathrm{Ni} @ \mathrm{SiO}_{2}$-yolk $)$ 不一 致, 说明光反应活性的增强不仅仅是 $\mathrm{Ni}$ 等离子体 效应引起的。进一步的热重分析(TGA)实验表明积 碳和石墨碳物种是反应稳定性的重要影响因素。 $\mathrm{Ni} / \mathrm{SiO}_{2}-\mathrm{Im}$ 催化剂上积碳含量最高, 因此其稳定性 最差, 而 $\mathrm{Ni} @ \mathrm{SiO}_{2}$-yolk与催化剂上积碳含量远低 于 $\mathrm{Ni} / \mathrm{SiO}_{2}-\mathrm{Im}$ 且石墨碳含量较高, 因此最稳定。此 外, 光照能够降低 $\mathrm{Ni}$ 基催化剂积碳含量, 进一步提 升催化剂稳定性。

\section{2 甲烷光催化制备含氧产物}

甲烷在氧化剂作用直接转化为醇、酸等含氧 产物近年来备受关注。由于甲烷氧化时易过度氧 化转变为 $\mathrm{CO}_{2}{ }^{46-48}$, 调控氧化程度尤为重要。甲烷 部分氧化(partial oxidation of methane, POM)是高 选择性制备含氧产物的重要途径, 常选用温和氧 化剂 $\left(\mathrm{H}_{2} \mathrm{O} 、 \mathrm{~N}_{2} \mathrm{O} \text { 等 }\right)^{49,50}$, 但仍存在甲烷转化率较低 的问题。因此, 发展高效的甲烷转化利用方法意义 重大。

$\mathrm{WO}_{3}$ 带宽为 $2.4-2.8 \mathrm{eV}$, 在酸性条件下于可见 光范围内具有稳定的光催化活性, 因此比较适合 于作为光催化剂 ${ }^{51,52}$ 。Villa等 ${ }^{21}$ 合成负载La的介孔 $\mathrm{WO}_{3}$ 用于光催化甲烷制甲醇反应。在该反应过程 中, $\mathrm{WO}_{3}$ 在光照条件下产生光生电子和空穴, $\mathrm{h}^{+}$与 吸附的水 $\left(\mathrm{H}_{2} \mathrm{O}_{\mathrm{ads}}\right)$ 和表面羟基 $\left(\mathrm{HO}_{\mathrm{ads}}^{-}\right)$发生反应产 生吸附的差基自由基 $\left(\mathrm{HO}_{\mathrm{ads}} \cdot\right)$, 随后活化甲烷以产 生甲基自由基 $\left(\mathrm{CH}_{3} \cdot\right)$, 进而偶联生成甲醇。此外, 掺杂 $\mathrm{La}$ 使 $\mathrm{WO}_{3}$ 在焙烧过程中保持结构并且增加了 其表面羟基自由基数量, 而表面羟基能与甲烷直 接反应生成甲醇, 因而活性大幅提升。Gondal等 ${ }^{53}$ 则采用等体积浸渍法在 $\mathrm{WO}_{3}$ 上负载 $\mathrm{Ag}$ 用于甲烷光 催化制甲醇。结果表明, $\mathrm{Ag}^{+}$与 $\mathrm{WO}_{3}$ 共享表面氧, 增 强其对光子的吸收能力并延长激发态寿命。 $\mathrm{Ag} /$ $\mathrm{WO}_{3}$ 外表面还可发生水解反应产生羟基自由基, 将 甲烷直接转化为甲醇。

$\mathrm{BiVO}_{4}$ 和 $\mathrm{V}_{2} \mathrm{O}_{5}$ 也被广泛应用于光氧化甲烷制 备含氧产物中 23,54 。Andreu等 ${ }^{33}$ 通过对 $\mathrm{NH}_{4}$ 型 $\beta$ 分子 笮 $\left(\mathrm{NH}_{4} \mathrm{BEA}\right)$ 进行改性制备了 $\mathrm{H}$ 型 $\beta$ 分子篮 (HBEA), 并利用等体积浸渍法制备Bi-V-HBEA催 化剂用于甲烷光催化制甲醇反应。X射线衍射 (XRD) 表明 $\mathrm{Bi}$ 能与 $\mathrm{V}$ 形成 $\mathrm{V}_{2} \mathrm{O}_{5} / \mathrm{BiVO}_{4}$ 异质结。如示 


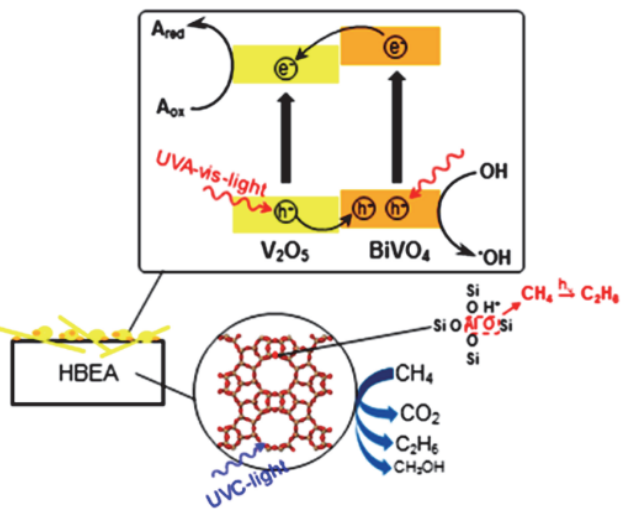

示意图1 Bi-V-HBEA光催化活化甲烷示意图

Scheme 1 Scheme of the photoactivation of different components in the Bi-V-HBEA photocatalyst.

Reproduced with permission from reference ${ }^{33}$.

意图1所示, 光照使 $\mathrm{V}-\mathrm{Bi}$ 异质结表面产生电子空穴 对, 并在 $\mathrm{V}_{2} \mathrm{O}_{5}$ 和 $\mathrm{BiVO}_{4}$ 之间传输, 提高了催化剂的 吸光能力与电子传输能力。 $\mathrm{h}^{+}$能够将表面羟基 $(\mathrm{OH})$ 氧化为表面羟基自由基 $(\cdot \mathrm{OH})$, 并与甲烷反应 生成甲基自由基 $\left(\cdot \mathrm{CH}_{3}\right)$ 。 $\mathrm{CH}_{3}$ 解吸后可与 $\mathrm{H}_{2} \mathrm{O}$ 结合 产生 $\mathrm{CH}_{3} \mathrm{OH}$, 也可自身偶联生成 $\mathrm{C}_{2} \mathrm{H}_{6}$ (框架中的

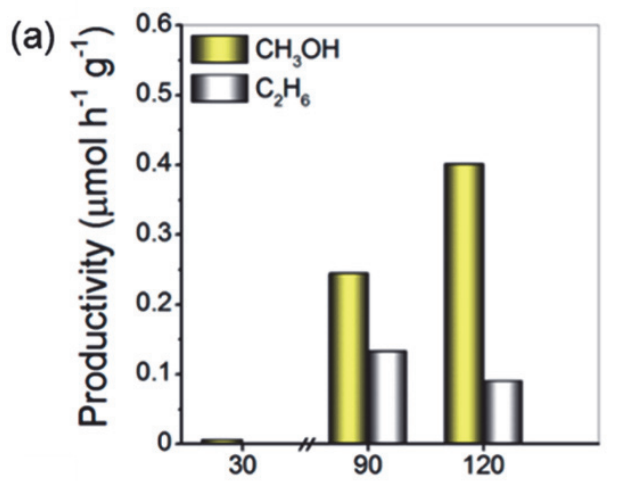

(b)

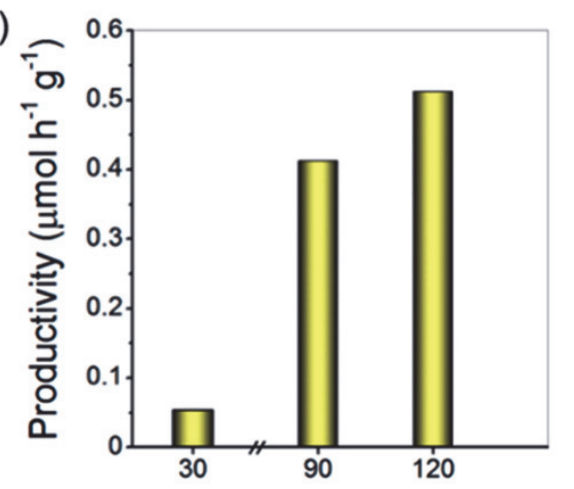

图4 (a) V-HBEA和(b) Bi-V-HBEA在不同时间 $(30,90$,

$120 \mathrm{~min}$ )下的甲醇和乙烷产率

Fig. $4 \mathrm{CH}_{3} \mathrm{OH}$ and $\mathrm{C}_{2} \mathrm{H}_{6}$ productivity obtained with

(a) V-HBEA and (b) Bi-V-HBEA at different times (30, 90, $120 \mathrm{~min})$.

Reproduced with permission from reference ${ }^{33}$.
$\mathrm{A} 1-\mathrm{O}$ 单元是偶联反应的活性位点)。从图 $4 \mathrm{a}, \mathrm{b}$ 中 可以看到, V-HBEA催化剂在 $30 \mathrm{~min}$ 时产物主要为 甲醇, 但是随着反应时间延长, 生成一定量的乙 烷。而Bi-V-HBEA催化剂无乙烷生成, 同时甲醇产 率有所提升。这是因为Bi降低了催化剂的布朗斯 特酸位点浓度, 从而表现出更高的甲醇选择性。此 外, 大比表面积的HBEA载体促进 $\mathrm{V}$ 分散, 增强了 吸光能力, 且其独特的孔道结构(孔道尺寸为 55 $\mathrm{nm}$ ) 有利于与甲烷 (动力学直径为 $3.8 \mathrm{~nm}$ ) 接触, 故 表现出较好的甲醇生成速率。

除固体催化剂外, 无机盐也可作为光催化剂。 Ohkubo等 ${ }^{55}$ 采用 $\mathrm{NaClO}_{2}$ 作为氧化剂, 在两相溶剂 $\mathrm{PFH}\left(n-\mathrm{CF}_{3}\left(\mathrm{CF}_{2}\right)_{4} \mathrm{CF}_{3}\right) / \mathrm{H}_{2} \mathrm{O}$ 中将甲烷直接转化为甲 醇和甲酸。常温常压下, 甲烷转化率高达 $99 \%$, 甲 醇选择性为 $14 \%$, 甲酸选择性为 $85 \%$ 。如示意图2 所示, $\mathrm{ClO}^{-}$在水相中与质子反应产生 $\mathrm{ClO}$ - 进入 $\mathrm{PFH}$ 有机相, 光照后 $\mathrm{Cl}-\mathrm{O}$ 键裂解产生 $\mathrm{Cl} \cdot$ 与 $\mathrm{O}_{2}, \mathrm{Cl}$. 氧化甲烷生成 $\mathrm{CH}_{3} \cdot(\mathrm{PFH}$ 由更强的 $\mathrm{C}-\mathrm{F}$ 键组成, 不 与自由基反应), 再与氧气反应产生甲醛或者甲 酸。此外, PFH对甲烷与氧气的溶解度高, 有利于 促进反应进行, 且对产物甲醛与甲酸溶解度低, 故 产物可以迅速转移到水相, 避免进一步氧化。

为了探究该过程的反应机理, 他们以 ${ }^{18} \mathrm{O}_{2}$ 做氧 同位素标记, GC-MS 测定数据显示产物为 $\mathrm{CH}_{3}{ }^{18} \mathrm{OH}$, 说明气相氧 ${ }^{18} \mathrm{O}_{2}$ 在反应中解离为 ${ }^{18} \mathrm{O}$ 并 参与甲醇的生成, 进一步证明产物氧来自于氧分 子; 以 $\mathrm{CD}_{4}$ 代替 $\mathrm{CH}_{4}, \mathrm{Cl}$ 不能使 $\mathrm{C}-\mathrm{D}$ 键断裂, 因此 没有含氧产物生成。他们还结合密度泛函理论 (DFT) 计算解释了甲醇与甲酸选择性差异主要取 决于 $\mathrm{A} 、 \mathrm{~B}$ 两个循环的反应活性。如示意图3所示, $\mathrm{B}$ 循环(生成甲酸)中 $\mathrm{CH}_{3} \mathrm{O}$ ·发生重排生成 $\mathrm{CH}_{2}(\mathrm{OH})$. 的 $\Delta E$ 为 $-15.23 \mathrm{~kJ} \cdot \mathrm{mol}^{-1}$, 而 $\mathrm{A}$ 循环 (生成甲醇) 中 $\mathrm{CH}_{3} \mathrm{O}$. 与甲烷反应生成 $\mathrm{CH}_{3}$. 的 $\Delta E$ 为 +5.44 $\mathrm{kJ} \cdot \mathrm{mol}^{-1}$, 故而甲酸选择性更高。值得注意的是,

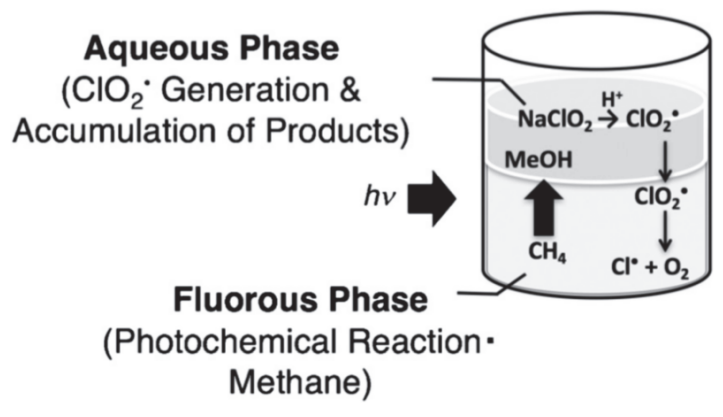

示意图2 两相溶液中 $\mathrm{NaClO}_{2}$ 光氧化 $\mathrm{CH}_{4}$ 示意图

Scheme 2 Two-phase photooxidation of $\mathrm{CH}_{4}$ by $\mathrm{NaClO}_{2}$.

Reproduced with permission from reference ${ }^{55}$ 


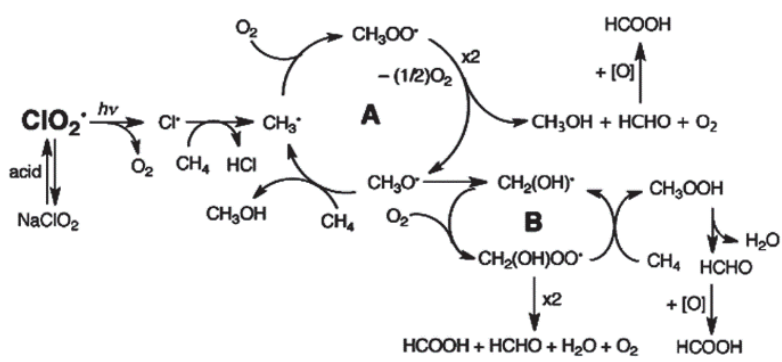

示意图3 $\mathrm{ClO}_{2}$.氧化甲烷形成甲醇和甲酸的反应机理

Scheme 3 Plausible reaction mechanism for oxygenation of $\mathrm{CH}_{4}$ into $\mathrm{CH}_{3} \mathrm{OH}$ and formic acid with $\mathrm{ClO}_{2}$.

Reproduced with permission from reference ${ }^{55}$.

$\mathrm{A}$ 循环中, $\mathrm{CH}_{3}$. 与 $\mathrm{O}_{2}$ 反应生成的 $\mathrm{CH}_{3} \mathrm{OO}$ 的 $\Delta E$ 为 $-154.07 \mathrm{~kJ} \cdot \mathrm{mol}^{-1}$, 因此 $\mathrm{A}$ 循环整体在热力学上也是 有利的。

\section{3 甲烷光催化偶联}

甲烷重整和选择性氧化反应一般只能将 $\mathrm{CH}_{4}$ 转化为其他 $\mathrm{C}_{1}$ 产物。如何通过有效手段实现碳链 增长进而制备 $\mathrm{C}_{2+}$ 产物是当今研究热点。甲烷偶联 (coupling of methane, COM)反应可以实现这一目 标。甲烷偶联起源于 19 世纪 80 年代, Keller和 Bhasin ${ }^{56}$ 发现在氧气气氛下甲烷可转化为 $\mathrm{C}_{2+}$ 烃类 $\left(>800^{\circ} \mathrm{C}\right)$ 。在该反应过程中, 甲烷先被活化为 $\mathrm{CH}_{x}$ 物种, 再二聚为 $\mathrm{C}_{2} \mathrm{H}_{y}$ 物种, 发生链增长或者异构化 生成 $\mathrm{C}_{2}+$ 烃类, 若与解离的氧原子作用则生成 $\mathrm{C}_{2}+$ 含 氧产物 ${ }^{57-59}$ 。偶联反应通常需要较高温度, 且由于 氧气存在, 易发生过度氧化并产生大量热力学稳 定的 $\mathrm{CO}_{2}$ 和 $\mathrm{H}_{2} \mathrm{O}$, 而通过光催化活化甲烷分子不仅 能够降低反应温度, 还能减少副产物生成。

甲烷可极化度低, 仅为 $2.84 \times 10^{-40} \mathrm{C}^{2} \cdot \mathrm{m}^{2} \cdot \mathrm{J}^{-1}$, 而Long等 37 制备了择优暴露 $\{001\}$ 晶面的 $\mathrm{ZnO}$ 纳 米片，并负载 $\mathrm{Au}$ 用于甲烷偶联制备乙烷反应。 $\mathrm{ZnO}\{001\}$ 晶面由于其极性较高 $\left(0.047 \mathrm{C} \cdot \mathrm{m}^{-2}\right)$, 能够 诱导其表层 $[\mathrm{ZnO}]$ 形成内电场, 提高对甲烷的亲和 力并促进光生电荷的分离。此外, $\mathrm{Au}$ 纳米粒子较 强的等离子体诱导效应产生电的磁场使得甲烷较 易在 $\mathrm{Au} / \mathrm{ZnO}$ 催化剂上活化生成 $* \mathrm{CH}_{4}$, 进一步与另 一分子 $\mathrm{CH}_{4}$ 偶联发生脱氢反应生成乙烷。单独 $\mathrm{Au}$ 纳米粒子和 $\mathrm{ZnO}$ 纳米片几乎无反应活性, 但是将 $\mathrm{Au}$ 负载于 $\mathrm{ZnO}$ 纳米片上后, 反应活性显著提高(图 $5)$, 说明 $\mathrm{Au}$ 与 $\mathrm{ZnO}$ 载体之间的界面起到至关重要 的作用。通过改变 $\mathrm{Au}$ 的负载量, 可调变乙烷的生 成速率。当 $\mathrm{Au}$ 负载量为 $4.8 \%$ 时, 乙烷和氢气产率 最高。

Chen等 ${ }^{38}$ 合成Zn修饰的ZSM-5 分子篎用于甲 烷光催化脱氢制备乙烷。在室温高压永灯辐射下,

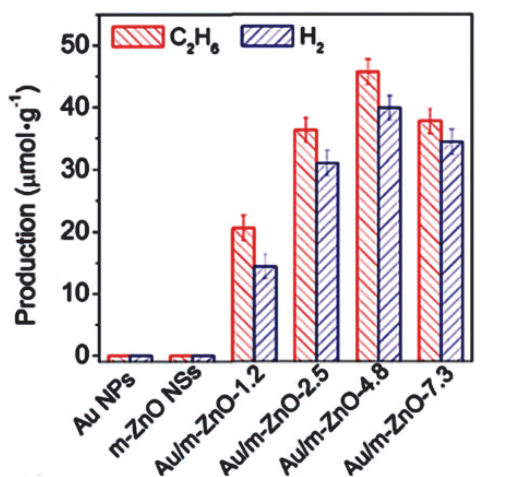

图5 光照 $4 \mathrm{~h}$ 后不同催化剂的甲烷偶联性能

Fig. 5 Comparison of photocatalytic methane coupling activity on different photocatalysts under solar light irradiation for $\mathbf{4 h}$.

Reproduced with permission from reference ${ }^{37}$.

(a)

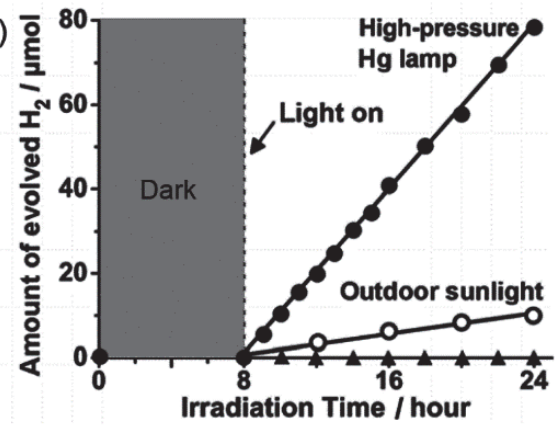

(b)

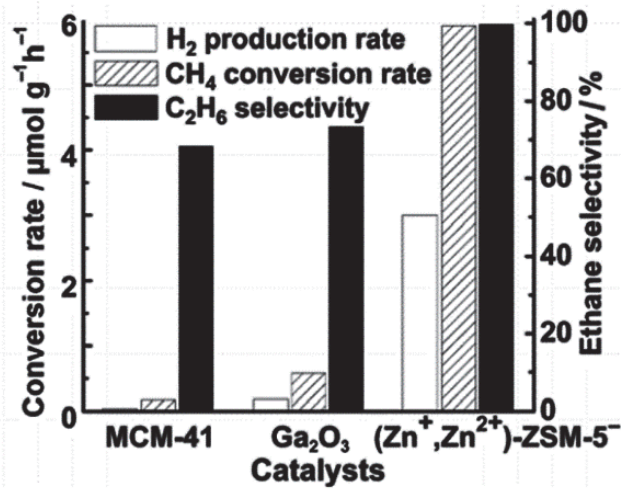

图6 (a)室温下甲烷在 $\left(\mathrm{Zn}^{+}, \mathrm{Zn}^{2+}\right)-\mathrm{ZSM-5}$ 催化剂上产

氢速率随时间变化曲线图, (b)不同光催化剂的 甲烷转化率、产氢速率及乙烷选择性

Fig. 6 (a) Photocatalytic hydrogen evolution as a function of time obtained at room temperature in the non-oxidative coupling of methane reaction catalyzed by $\left(\mathrm{Zn}^{+}, \mathrm{Zn}^{2+}\right)$ -

$\mathrm{ZSM}^{-}$, (b) Methane conversion rate, hydrogen production rate and ethane selectivity obtained for NOCM reaction catalyzed by different catalysts. Reproduced with permission from reference ${ }^{38}$.

$8 \mathrm{~h}$ 内甲烷转化率达到 $24 \%$ ，乙烷选择性大于 $99 \%$ 。 如图 $6 a, b$ 所示, 光照显著提高 $\mathrm{H}_{2}$ 产率, 且以高压 永灯作为光源时有最佳的产氢速率。 $\left(\mathrm{Zn}^{+}, \mathrm{Zn}^{2+}\right)$ - 
ZSM-5催化剂上甲烷反应速率达到 $6 \mu \mathrm{mol} \cdot \mathrm{h}^{-1} \cdot \mathrm{g}^{-1}$, 乙烷选择性接近 $100 \%$, 远高于 $\mathrm{Ga}_{2} \mathrm{O}_{3}(0.58$ $\left.\mu \mathrm{mol} \cdot \mathrm{h}^{-1} \cdot \mathrm{g}^{-1}\right)$ 和MCM-41 $\left(0.17 \mu \mathrm{mol} \cdot \mathrm{h}^{-1} \cdot \mathrm{g}^{-1}\right)$ 。理论 计算和电子顺磁共振 (EPR)结果表明, 光照下 ZSM-5沸石框架内 $\mathrm{Zn}^{+}$的 $4 s$ 轨道能够释放价电子活 化甲烷产生 $\mathrm{CH}_{4}{ }^{*}$, 同时 $\mathrm{Zn}^{+}$被氧化为 $\mathrm{Zn}^{2+}$, 活化的 $\mathrm{CH}_{4} *$ 与另一分子 $\mathrm{CH}_{4}$ 反应生成乙烷 (示意图4)。此 外, 由于 $\left(\mathrm{Zn}^{+}, \mathrm{Zn}^{2+}\right)-\mathrm{ZSM}-5$ 孔道大小限制(孔道大 小为 $0.55 \mathrm{~nm})$, 不足以让两个乙烷分子在 $\mathrm{Zn}^{+}$活性 位点上发生偶联反应生成丁烷, 故而后续反应被 切断, 从而体现出极高的乙烷选择性。

\section{4 基于配体到金属电子跃迁途径的甲烷光催 化转化}

除了以上经典的用于甲烷光活化手段之外, 光 诱导电子跃迁产生自由基进一步直接活化甲烷的 方法也逐渐进入人们的视野, 其中以配体到金属 电子跃迁 (LMCT) 途径研究较为广泛。配体到金属 电子跃迁途径是指当配合物被激发时, 配体的 $\pi$ 电 子跃迁到金属上的 $d$ 轨道形成激发态成为 LMCT 态。在LMCT途径中, 光照后即诱发配位键均裂形 成有机自由基物种, 减少中间态从而能最大限度 利用光能来实现有机分子的单电子氧化。对于具 有高氧化电势的化合物, 如醇类化合物, LMCT是 一种直接形成高能烷氧自由基的高效活化手段。

基于LMCT途径能够直接形成高能烷氧自由 基的特点, 左智伟课题组在醇类功能化、甲烷活化 等领域开展系统性工作 ${ }^{60-62}$ 。该小组首次在 LED蓝 光作用下利用简单、廉价易得的 $\mathrm{CeCl}_{3}$ 催化剂用于 环烷醇的 $\mathrm{C}-\mathrm{C}$ 键断裂-氨化反应, 产物选择性高达 $92 \%$, 且该过程适用于各种仲醇的功能化 ${ }^{60}$ 。随后

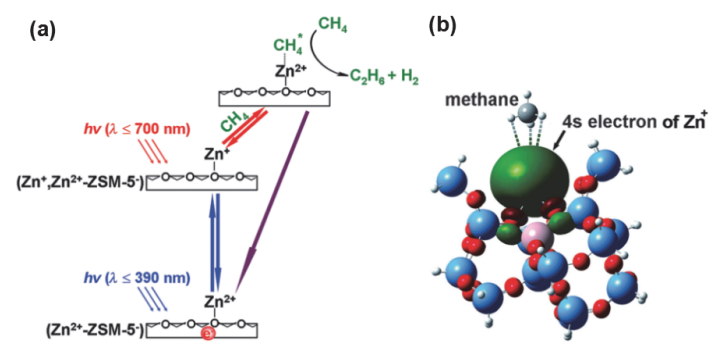

示意图4 (a)光催化反应示意图; (b) $\mathrm{Zn}^{+}$活性位点与 甲烷分子作用形成的B3LYT杂化交换关联优化几何结构

Scheme 4 (a) Schematic energy diagram for the processes of the photocatalytic reaction and (b) the B3LYT hybrid exchange-correlation optimized geometry of the adsorbed methane molecule attracted by the $\mathrm{Zn}^{+}$active site

Red: O, pink : Si, gray: C, white: $\mathrm{H}$, and green: the $4 s$ electron of $\mathrm{Zn}^{+}$.

Color online. Reproduced with permission from reference ${ }^{38}$.
该小组又将 $\mathrm{LMCT}$ 催化剂体系拓展到伯醇功能化 体系中, 成功实现对碳链上的 $\delta$ 位功能化。研究发 现, LED蓝光作用下, 伯醇可以产生烷氧自由基, 烷氧自由基的电子与 $\delta$ 位的氢发生位置互换, 并在 $\delta$ 位生成自由基, 提供功能化位点, 从而实现对 $\delta$ 位 $\mathrm{C}-\mathrm{H}$ 键活化 ${ }^{61}$ 。最近, 该小组成功将 $\mathrm{Ce}$ 基光催化 剂LMCT途径应用于甲烷的光催化活化 ${ }^{62}$ 。如示意 图 5所示, 以三氟甲磺酸铈和三氯乙醇作为催化 剂, 在LED蓝光照射下, 三氯乙醇会产生烷氧高能 自由基。该自由基可以通过氢转移途径活化甲烷 $\mathrm{C}-\mathrm{H}$ 键并形成相应的甲基自由基，再与自由基捕 获剂发生偶联反应生成甲基肼, 同时铈基催化剂 也完成一个催化循环, 实现甲烷高效、高选择性的 烷基化和芳基化。此外, 该方法还可拓展到其它烃 类及多种碳氢键的活化转化。

\section{3 甲醇光催化转化}

甲醇是重要的化工原料, 可用来制备甲醛、二 甲醚、乙酸、甲基叔丁基醚、二甲基甲酰胺和甲胺 等上百种化工产品, 因此甲醇高效转化一直颇受 关注 ${ }^{63}$ 。目前, 国内煤基制备甲醇工艺不断成熟, 甲醇产能过剩。因此, 将其转化为下游高附加值化 工产品能促进甲醇行业的健康发展。

传统热催化反应倾向于活化甲醇 $\mathrm{O}-\mathrm{H}$ 键而 非 $\mathrm{C}-\mathrm{H}$ 键, 且活化 $\mathrm{C}-\mathrm{H}$ 键则需要更高的温度。而 光催化对 $\mathrm{C}-\mathrm{H}$ 键有独特的选择性, 能够使 $\mathrm{C}-\mathrm{H}$ 键 均裂产生高能自由基, 不仅能够实现温和条件下 的甲醇转化, 还能实现 $\mathrm{C}-\mathrm{C}$ 偶联反应。本节将从 光催化角度介绍甲醇重整、氧化和偶联三种反应

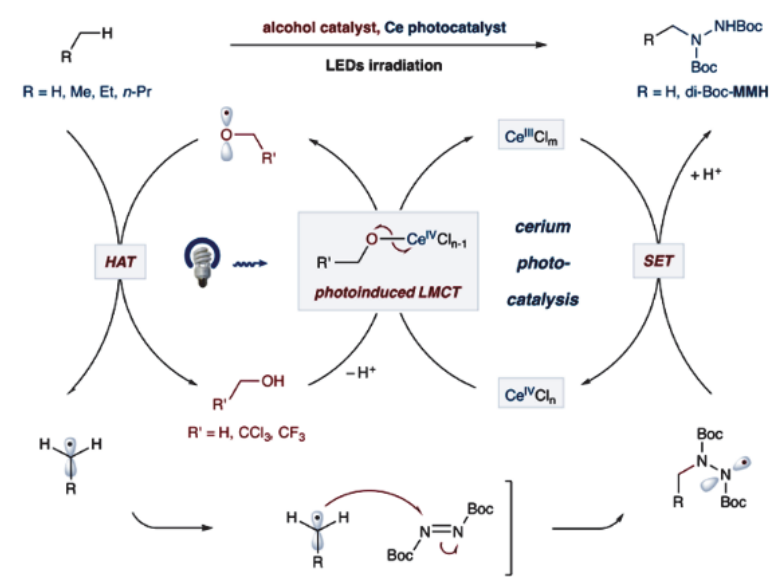

示意图5 铈催化剂催化甲烷和其它烷烃 $C\left(s p^{3}\right)-H$ 活化反应机理

Scheme 5 Proposed mechanism for the cerium-catalyzed $\mathrm{C}\left(s \mathrm{p}^{3}\right)-\mathrm{H}$ functionalization of methane and other alkanes.

Reproduced with permission from reference ${ }^{62}$. 
表2 各类甲醇光催化反应催化性能的汇总

Table 2 Catalytic performances for methanol conversion over different photocatalysts.

\begin{tabular}{|c|c|c|c|c|c|c|c|c|c|c|}
\hline \multirow{2}{*}{ Entry } & \multirow{2}{*}{ Catalyst } & \multirow{2}{*}{ Reaction } & \multicolumn{4}{|c|}{ Reaction conditions } & \multicolumn{3}{|c|}{ Catalytic performance } & \multirow{2}{*}{ Ref } \\
\hline & & & $T /{ }^{\circ} \mathrm{C}$ & $P / \mathrm{MPa}$ & Light source & $I /\left(\mathrm{mW} \cdot \mathrm{cm}^{-2}\right)$ & Reaction rate & Conv. $/ \%$ & Sel./\% & \\
\hline 1 & $0.5 \% \mathrm{Pt} / \mathrm{TiO}_{2}$ & $\begin{array}{l}\text { methanol to } \\
\text { hydrogen }\end{array}$ & RT & 0.1 & LED (365 nm) & 3 & $66.75 \mathrm{~mol}_{\mathrm{H}_{2}} \cdot \mathrm{g}^{-1} \cdot \mathrm{min}^{-1}$ & NA & NA & 22 \\
\hline 2 & $1 \% \mathrm{Pt} / \mathrm{TiO}_{2}$ & $\begin{array}{l}\text { methanol to } \\
\text { hydrogen }\end{array}$ & 30 & 0.1 & $\begin{array}{l}\text { iron halogenide } \\
\text { mercury arc lamp }\end{array}$ & $\begin{array}{l}1.67 \times 10^{-7} \\
\text { Einsteins }{ }^{-1} \cdot \mathrm{cm}^{-2}\end{array}$ & $\begin{array}{l}18.6 \mathrm{mmol}_{\mathrm{H}_{2}} \cdot \mathrm{g}^{-1} \cdot \mathrm{h}^{-1} \\
2.88 \mathrm{mmol}_{\mathrm{CO}_{2}} \cdot \mathrm{g}^{-1} \cdot \mathrm{h}^{-1}\end{array}$ & NA & $\begin{array}{l}\mathrm{CO}_{2}: \\
46.4\end{array}$ & 64 \\
\hline 3 & $\mathrm{MgO}$ & $\begin{array}{l}\text { methanol to } \\
\text { formaldehyde }\end{array}$ & RT & 0.1 & mercury lamp UV & 388 & $\sim 55 \mu \mathrm{mol}_{\mathrm{H}_{2}} \cdot \mathrm{g}^{-1} \cdot \mathrm{h}^{-1}$ & NA & no $\mathrm{CO}_{x}$ & 65 \\
\hline 4 & $\begin{array}{c}\mathrm{AgNPs} / \mathrm{g}-\mathrm{C}_{3} \mathrm{~N}_{4} \\
\text { aerogel }\end{array}$ & $\begin{array}{l}\text { methanol to } \\
\text { formaldehyde }\end{array}$ & RT & 0.1 & $\begin{array}{c}\text { Xe lamp } \\
(350-780 \mathrm{~nm})\end{array}$ & NA & $152.2 \mu \mathrm{mol}_{\mathrm{H}_{2}} \cdot \mathrm{g}^{-1} \cdot \mathrm{h}^{-1}$ & NA & no $\mathrm{CO}_{x}$ & 66 \\
\hline 5 & $\mathrm{Au} / \mathrm{WO}_{3}$ & $\begin{array}{l}\text { methanol to } \\
\text { formaldehyde }\end{array}$ & 25 & 0.1 & $\begin{array}{l}\text { Xe arc lamp } \\
(380-950 \mathrm{~nm})\end{array}$ & 800 & $120 \mu \mathrm{mol}_{\mathrm{MeOH}} \cdot \mathrm{g}^{-1} \cdot \mathrm{min}^{-1}$ & NA & $32-40$ & 67 \\
\hline 6 & $\mathrm{Cu} / \mathrm{TiO}_{2}$ & $\begin{array}{c}\text { methanol to } \\
\text { methyl formate }\end{array}$ & $15-45$ & 0.1 & $\begin{array}{c}\text { high pressure } \\
\text { mercury lamp UV }\end{array}$ & 18.6 & $56.4 \mathrm{mmol}_{\mathrm{MF}} \cdot \mathrm{g}^{-1} \cdot \mathrm{h}^{-1}$ & $65-85$ & $55-65$ & 68 \\
\hline 7 & $\begin{array}{c}\mathrm{CuO} / \mathrm{CuZnAl} \\
\text { hydrotalcites- } \\
\mathrm{ZnO}\end{array}$ & $\begin{array}{l}\text { methanol to } \\
\text { methyl formate }\end{array}$ & $26-45$ & 0.1 & $\begin{array}{c}\text { high pressure } \\
\text { mercury lamp UV }\end{array}$ & 25.3 & NA & 84.5 & 59.1 & 69 \\
\hline 8 & $3 \% \mathrm{Ag} / \mathrm{SiO}_{2}$ & $\begin{array}{l}\text { methanol to } \\
\text { methyl formate }\end{array}$ & 40 & 0.1 & $\begin{array}{c}\text { high pressure } \\
\text { mercury lamp UV }\end{array}$ & NA & $23.46 \mathrm{mmol}_{\mathrm{MF}} \cdot \mathrm{g}^{-1} \cdot \mathrm{h}^{-1}$ & NA & NA & 70 \\
\hline 9 & $\mathrm{Au}-\mathrm{Ag}$ alloy/ $\mathrm{TiO}_{2}$ & $\begin{array}{l}\text { methanol to } \\
\text { methyl formate }\end{array}$ & $15-45$ & 0.1 & UV & $\sim 19$ & $\sim 22 \mathrm{mmol}_{\mathrm{MF}} \cdot \mathrm{g}^{-1} \cdot \mathrm{h}^{-1}$ & 90 & 85 & 71 \\
\hline 10 & $\mathrm{MoS}_{2}$ foam/CdS & $\begin{array}{l}\text { methanol to } \\
\text { ethylene glycol }\end{array}$ & RT & 0.1 & $\begin{array}{l}\text { 300-W Xe lamp } \\
(420-780 \mathrm{~nm})\end{array}$ & NA & $\begin{array}{l}11 \mathrm{mmol}_{\mathrm{EG}} \cdot \mathrm{g}^{-1} \cdot \mathrm{h}^{-1} \\
12 \mathrm{mmol}_{\mathrm{H}_{2}} \cdot \mathrm{g}^{-1} \cdot \mathrm{h}^{-1}\end{array}$ & NA & 90 & 20 \\
\hline 11 & $\mathrm{Pt} / \mathrm{TiO}_{2}$ & $\begin{array}{l}\text { methanol to } \\
\text { ethylene glycol }\end{array}$ & 60 & 0.1 & $\begin{array}{l}\text { Xe lamp UV light } \\
(320-400 \mathrm{~nm})\end{array}$ & 120 & $16.8 \mathrm{umol}_{\mathrm{EG}} \cdot \mathrm{g}^{-1} \cdot \mathrm{h}^{-1}$ & NA & 86.4 & 72 \\
\hline 12 & $\mathrm{Pt} / \mathrm{TiO}_{2}$ & $\begin{array}{l}\text { methanol to } \\
\text { aliphatic alcohol }\end{array}$ & 60 & 0.1 & $\begin{array}{l}\text { Xe lamp irradiation } \\
\quad(200-400 \mathrm{~nm})\end{array}$ & 100 & $\begin{array}{l}\text { TOF of aliphatic } \\
\text { alcohol } 0.003 \mathrm{~h}^{-1}\end{array}$ & $1.3-8.6$ & $54-87$ & 73 \\
\hline
\end{tabular}

的研究进展(表2) 22,64-73。

\section{1 甲醇光催化重整制氢气}

氢气不仅是清洁能源, 同时也是工业生产中 的重要原料, 需求量巨大 74 。工业上大规模制氢有 两种方法: 一是在 $1.5-4 \mathrm{MPa}, 650-950{ }^{\circ} \mathrm{C}$ 的反应 条件下, 利用镍基催化剂重整天然气和水制备氢 气; 二是以水和煤为原料, 制备水煤气, 再通过 水煤气变换制取氢气。相对于目前工业上采用 的氢气制备方法, 甲醇与水重整制氢 $\left(\mathrm{CH}_{3} \mathrm{OH}+\right.$ $\mathrm{H}_{2} \mathrm{O} \rightarrow 3 \mathrm{H}_{2}+\mathrm{CO}_{2}$ ) 是一步反应, 并可将反应温度 降至到 $240-260{ }^{\circ} \mathrm{C}$, 在生产成本上具有极大优势, 且光催化能将温度进一步降到 $100^{\circ} \mathrm{C}$, 因而更具吸 引力。

Nomikos 等 ${ }^{22}$ 研究 $\mathrm{Pt} / \mathrm{TiO}_{2}$ 催化剂在紫外光下 甲醇重整的动力学和反应机理。实验结果显示, 0.37 $\mathrm{mmol} \cdot \mathrm{L}^{-1}$ 甲醇水溶液的产氢速率是纯水产氢速率
的6倍, 且随着甲醇不断被消耗, 产氢速率也不断 减少, 这表明甲醇是氢气的主要来源。气相色谱 (GC)、气相色谱-质谱联用仪(GC-MS)和漫反射傅 立叶变换红外光谱(DRIFTS)表征结果表明反应机 理(式(1)-(7))为: 甲醇吸附在二氧化钛上后分解成 $\mathrm{CH}_{3} \mathrm{O}_{(\mathrm{a})}^{-}$(吸附态)和 $\mathrm{H}^{+}, \mathrm{CH}_{3} \mathrm{O}_{(\mathrm{a})}^{-}$与空穴反应产生 $\mathrm{CH}_{3} \mathrm{O}_{(\mathrm{a})} \cdot$, 随后 $\mathrm{CH}_{3} \mathrm{O}_{(\mathrm{a})}$. 与空穴反应产生甲醛和质 子, 质子得到光生电子产生氢气。若甲醛与表面氧 和空穴进一步反应, 则继续释放质子得到甲酸、二 氧化碳等产物。Highfield等 ${ }^{75}$ 也提出相似的机理, 认为甲醇作为空穴消除剂, 逐步氧化后形成甲醛、 甲酸及二氧化碳, 并伴随着氢质子的释放。

$$
\begin{aligned}
& \mathrm{CH}_{3} \mathrm{OH} \rightarrow \mathrm{CH}_{3} \mathrm{O}_{(\mathrm{a})}^{-}+\mathrm{H}^{+} \\
& \mathrm{CH}_{3} \mathrm{O}_{(\mathrm{a})}^{-}+\mathrm{h}^{+} \rightarrow \mathrm{CH}_{3} \mathrm{O}_{(\mathrm{a})} \\
& \mathrm{e}^{-}+\mathrm{H}^{+} \rightarrow 1 / 2 \mathrm{H}_{2} \\
& \mathrm{CH}_{3} \mathrm{O}_{(\mathrm{a})}+\mathrm{h}^{+} \rightarrow \mathrm{CH}_{2} \mathrm{O}_{(\mathrm{a})}+\mathrm{H}^{+}
\end{aligned}
$$




$$
\begin{aligned}
& \mathrm{CH}_{2} \mathrm{O}_{(\mathrm{a})}+\mathrm{O}_{(\mathrm{s})} \rightarrow \mathrm{H}_{2} \mathrm{CO}_{2(\mathrm{a})} \\
& \mathrm{H}_{2} \mathrm{CO}_{2(\mathrm{a})}+\mathrm{h}^{+} \rightarrow \mathrm{HCOO}_{(\mathrm{a})}+\mathrm{H}^{+} \\
& \mathrm{HCOO}_{(\mathrm{a})}+\mathrm{h}^{+} \rightarrow \mathrm{H}^{+}+\mathrm{CO}_{2}
\end{aligned}
$$

为了探索贵金属在甲醇重整中的作用, Selli 课题组 ${ }^{76}$ 采用浸渍法制备 $\mathrm{Pt} / \mathrm{TiO}_{2}$ 与 $\mathrm{Au} / \mathrm{TiO}_{2}$ 催化 剂。ESR 结果表明, $\mathrm{Pt} / \mathrm{TiO}_{2}$ 上 $\mathrm{Ti}^{3+}$ 浓度最低, 即电 子更容易从 $\mathrm{Ti}^{3+}$ 转移到 $\mathrm{Pt}$ 纳米粒子上, 继而与 $\mathrm{Pt}$ 表 面的质子反应析出氢气。此外, HRTEM结果表明 负载的贵金属粒径越小, 光生载流子分离越快, 越 有利于反应进行。此外, 该小组 ${ }^{64}$ 采用火焰喷雾热 解法和沉积法制备不同贵金属负载的 $\mathrm{TiO}_{2}$ 催化 剂。结果也表明 $\mathrm{Pt}$ 是最有效的贵金属助剂, 其次是 金、银。

\section{2 甲醇光催化氧化制备高值化学品}

在众多甲醇下游产品中, 甲酫是用途非常广 泛的化工原料, 它是制造树脂、塑料、油漆、防皱 纺织品等材料的常见前体; 而甲酸甲酯被誉为 “万 能中间体”, 它同时具有甲酰基和酯基, 可进行多 种反应。本部分将介绍甲醇光催化制备甲醛和甲 酸甲酯的研究进展。

\subsection{1 甲醇光催化制备甲醛}

甲醛作为重要的化学中间体, 引起人们的广 泛关注。目前 $35 \%$ 的工业甲醇被用来制造甲酫 ${ }^{63}$, 但 是工业上该过程反应条件苛刻且原子经济性不 高。甲醇直接脱氢产生醛和氢气不但体现出很高 的原子经济性, 还可避免催化剂积碳失活, 有利于 延长催化剂的使用寿命。

甲醇存在 $\mathrm{C}-\mathrm{H}$ 键与 $\mathrm{O}-\mathrm{H}$ 键, 判断 $\mathrm{H}_{2}$ 来源对研 究氧化机理十分重要。Guo 等 77 发现光照下的 $\mathrm{CD}_{3} \mathrm{OH}$ 在 $\mathrm{TiO}_{2}(110)$ 晶面分解为吸附在 $\mathrm{Ti}_{5 \mathrm{c}}$ 位点上 的 $\mathrm{CD}_{2} \mathrm{O}$ 以及吸附在桥联 $\mathrm{O}_{\mathrm{br}}$ 上 $\mathrm{D} 、 \mathrm{H}$, 从而证实甲 醇光氧化是经历 $\mathrm{O}-\mathrm{H}$ 与 $\mathrm{C}-\mathrm{H}$ 两步裂解而得到甲 醛。Shen等 ${ }^{78}$ 进一步研究发现甲醇吸附在 $\mathrm{TiO}_{2}(110)$ 晶面上后, 首先热裂解 $\mathrm{O}-\mathrm{H}$ 键生成甲氧基和表面 羟基, 随后甲氧基光裂解 $\mathrm{C}-\mathrm{H}$ 键产生甲醛, 并且 认为决速步骤是甲醇热解为甲氧基。Z Zhang 等 ${ }^{79}$ 通 过DFT $+\mathrm{U}$ 理论计算研究证实甲醇的 $\mathrm{O}-\mathrm{H}$ 是由热 引发的异裂, $\mathrm{C}-\mathrm{H}$ 是由光催化引发的均裂, 如示 意图6所示。此外, 他们还探讨了光生空穴在 $\mathrm{C}-\mathrm{H}$ 裂解中的作用, 认为 $\mathrm{CH}_{3} \mathrm{O}^{-}$被空穴捕获后生成弱吸 附的 $\mathrm{CH}_{3} \mathrm{O}$, 使 $\mathrm{Ti}-\mathrm{OCH}_{3}$ 结合能下降, 能垒降低, 从而促进甲醛的解吸。

通过对催化剂进行结构修饰能显著提高催化 性能。Yin等 ${ }^{65}$ 通过绿色简便的热解法制备了尺寸 可调控 (40-170 nm) 的 $\mathrm{MgO}$ 多孔纳米颗粒, 发现利 用氧等离子体处理后的催化剂亲水性更好, 在极

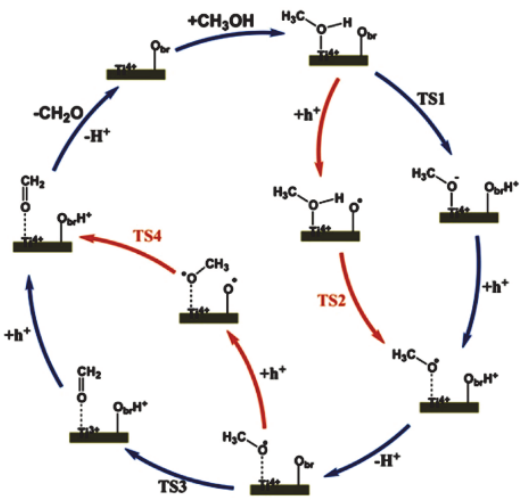

示意图6 甲醇解离的多种路径

Scheme 6 Illustration of various reaction pathways for the dissociation of methanol.

The pathways marked in blue are the most preferred pathways. Color online. Reproduced with permission from reference ${ }^{79}$.

性溶剂中更易分散。当催化剂浓度为 0.38 $\mathrm{mg} \cdot \mathrm{mL}^{-1}$, 粒径为 $85 \mathrm{~nm}$ 时, 在室温紫外光照下产 氢速率可达 $55 \mu \mathrm{mol} \cdot \mathrm{g}^{-1} \cdot \mathrm{h}^{-1}$ 。而 Yang 等 ${ }^{66}$ 以原位自 组装的方法制备了纤维结构的 $\mathrm{Ag} \mathrm{NPs} / \mathrm{g}-\mathrm{C}_{3} \mathrm{~N}_{4}$ 气凝 胶催化剂, 将反应的响应波长拓展到可见光。研究 发现该催化剂具有中等的氧化还原能力, 能够选 择性氧化甲醇为甲醛和氢气而不产生 $\mathrm{CO}_{x}$, 并且 $\mathrm{Ag}$ 纳米颗粒能以增强等离子共振的方式提高光催 化活性。

\subsection{2甲醇氧化制备甲酸甲酯}

甲酸甲酯(MF)是常见的低沸点溶剂, 也是有 效的杀虫剂、杀菌剂。工业上主要通过甲醇羰基化 制备 MF，在该过程中，干燥脱水的甲醇与 $\mathrm{CO}$ 在 $80^{\circ} \mathrm{C} 、 4 \mathrm{MPa}$ 下以甲醇钠为催化剂合成甲酸甲酯， 虽然产率高, 但操作成本高、污染大 ${ }^{80-82}$ 。甲醇直 接脱氢制MF由于受到热力学平衡限制, 产率一直 较低，但因其具有原料简单、成本低、污染小的特 点而受到关注 ${ }^{83}$ 。而光催化甲醇直接脱氢制备MF 可在低温常压下进行, 避免高温下甲酸甲酯的分 解, 摆脱热力学限制, 为甲醇直接脱氢制备MF提 供新的转机。

Phillips 等 ${ }^{84}$ 用质谱和扫描隧道显微镜证明在 $\mathrm{TiO}_{2}(110)$ 面上甲醇制备甲酸甲酯经过两个连续的 光氧化步骤。如示意图7所示, 甲醇 $\mathrm{O}-\mathrm{H}$ 键首先热 解离形成吸附的甲氧基, 被光生空穴氧化生成甲 醛, 接着甲醛进一步光氧化产生瞬态 $\mathrm{HCO}$, 与表面 残留甲氧基结合得到甲酸甲酯。在反应过程中, 二 氧化钛表面需要暴露于 $\mathrm{O}_{2}$ 中以修复表面和近表面 缺陷, 从而维持活性。

$\mathrm{Liu}$ 等 ${ }^{68}$ 制备 $\mathrm{Cu} / \mathrm{TiO}_{2}$ 催化剂并提出了类似机 理, 并解释 $\mathrm{Cu}$ 的作用。将 $\mathrm{Cu}$ 还原至金属态, 可增 


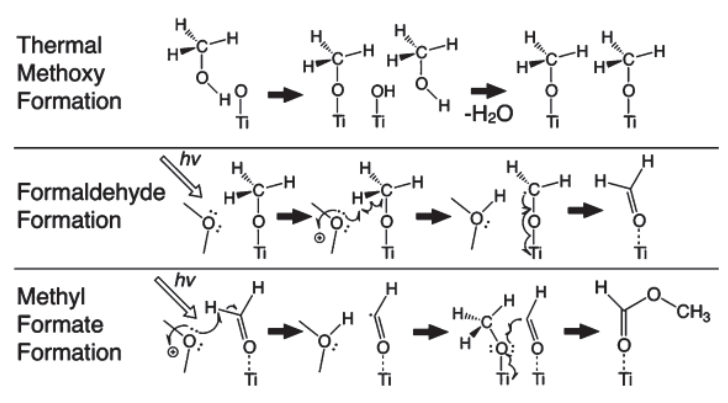

示意图7 甲醇在 $\mathrm{TiO}_{2}(110)$ 面上氧化制备甲酸甲酯的 催化机理

Scheme 7 Mechanism of the oxidation of methanol to methyl formate on $\mathrm{TiO}_{2}(110)$. Reproduced with permission from reference ${ }^{84}$.

强 $\mathrm{Cu}$ 与 $\mathrm{TiO}_{2}$ 之间的电子耦合, 从而促进光生载流 子分离。同时, 缺电荷的 $\mathrm{Cu}$ 表面有助于 $\mathrm{O}_{2}$ 解离为 $\mathrm{O}$ 原子, 溢出至 $\mathrm{TiO}_{2}$ 氧空位 $\left({ }^{\circ} \mathrm{S}\right)$ 上, 填补缺陷(如式 (8)-(10)所示)。在 $15-45^{\circ} \mathrm{C}$, 紫外光照射下, $M F$ 产 率可达 $56.4 \mathrm{mmol} \cdot \mathrm{g}^{-1} \cdot \mathrm{h}^{-1}$, 且 $30^{\circ} \mathrm{C}$ 以下副产物仅有 $\mathrm{CO}_{2}$ 。

$$
\begin{aligned}
& \mathrm{O}_{2}+\mathrm{e}^{-} \rightarrow 2 \mathrm{O}-\mathrm{Cu} \\
& \mathrm{O}_{2}^{-}-\mathrm{Cu}+\mathrm{e}^{-} \stackrel{\text { Dissociation }}{\longrightarrow} 2 \mathrm{O}-\mathrm{Cu} \\
& \mathrm{O}-\mathrm{Cu}+{ }^{\circ} \mathrm{S} \stackrel{\text { Spillover }}{\longrightarrow} \mathrm{Cu}+\mathrm{O}-\mathrm{S}
\end{aligned}
$$

通过结构修饰可以使本身无光催化活性的物 质产生活性。Liang 等 69 制 备 $\mathrm{CuO} / \mathrm{CuZnAl}$ hydrotalcites- $\mathrm{ZnO}$ 催化剂, 在 $26-45{ }^{\circ} \mathrm{C}$ 及紫外光照 射下可实现甲醇氧化制备 $\mathrm{MF}$ 。在 $30{ }^{\circ} \mathrm{C}$ 时, $\mathrm{MF}$ 产 率最大可达 $50 \%$, 且副产物仅有 $\mathrm{CO}_{2}$ 。尽管 $\mathrm{CuZnAl}$ 水滑石和 $\mathrm{ZnO}$ 都对此反应没有活性, 但两者接触界 面不仅能提供羟基, 还能在光照下发生能带弯曲 产生具有氧化能力的空穴, 推动反应进行。氧化铜 在反应中能捕获导带电子并解离化学吸附的氧分 子, 在反应结束后转化为金属态。

Yang 等 ${ }^{70}$ 制备了 $\mathrm{Ag} / \mathrm{SiO}_{2}$ 和 $\mathrm{Ag} / \mathrm{TiO}_{2}(\mathrm{P} 25)$ 催化 剂, 在紫外光照射下均可选择性氧化甲醇为甲酸 甲酯(图7), 其中 $A g$ 有极其重要的作用。光照下, 在 $\mathrm{SiO}_{2}$ 表面上, $\mathrm{Ag}$ 纳米粒子产生等离子体共振效应, $10 \mathrm{~h}$ 内 MF生成速率可达 $23460 \mu \mathrm{mol} \cdot \mathrm{g}^{-1} \cdot \mathrm{h}^{-1}$ 。在 $\mathrm{TiO}_{2}$ 表面上, $\mathrm{Ag}$ 纳米粒子捕获光生电子并延长空 穴电子寿命, 将MF生产速率提高20倍。

\section{3 基于甲醇光催化偶联的羟甲基化反应}

羟甲基在天然产物和生物活性分子中广泛存 在, 可经多种合成转化过程得到不同的衍生物, 在 有机合成中具有重要意义。甲醇作为 $\mathrm{C}_{1}$ 醇, 是着弪甲 基化反应最简单直接的来源, 活化 $\mathrm{C}-\mathrm{H}$ 键即可产 生羟甲基自由基, 能自身偶联或与含不饱和键的 化合物(如醛、酮和烯烃等) $\mathrm{C}-\mathrm{C}$ 偶联发生着圣甲基 (a)

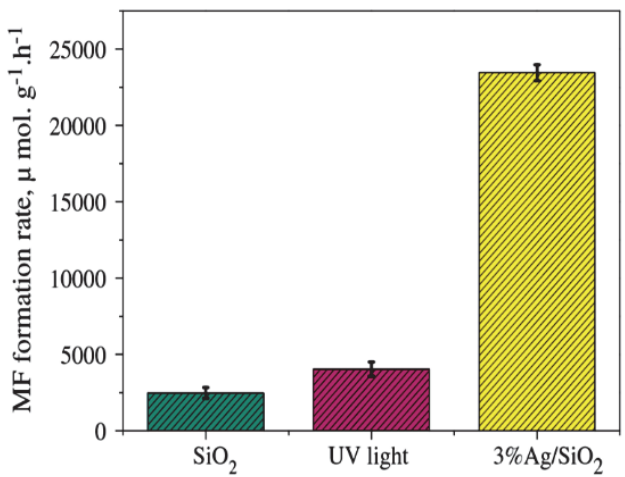

(b)

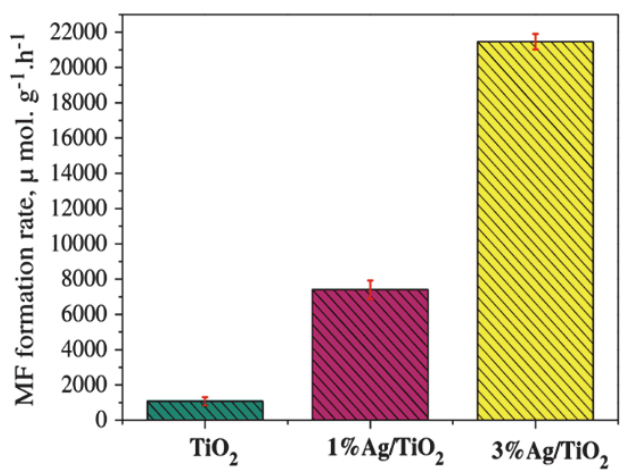

图7 (a)甲酸甲酯在 $\mathrm{SiO}_{2} 、 \mathrm{UV}$ 光照(无催化剂)和 $\mathrm{Ag} / \mathrm{SiO}_{2}$ 上的生成速率，(b)甲酸甲酯在 $\mathrm{TiO}_{2}$ 、

$1 \% \mathrm{Ag} / \mathrm{TiO}_{2}$ 和 $3 \% \mathrm{Ag} / \mathrm{TiO}_{2}$ 上的生成速率

Fig. 7 Production of methyl formate during reaction

(a) of silica, UV light (without catalyst) and silver catalysts supported silica and (b) of $\mathrm{TiO}_{2}$, $1 \% \mathrm{Ag} / \mathrm{TiO}_{2}$ and $3 \% \mathrm{Ag} / \mathrm{TiO}_{2}$. Reproduced with permission from reference ${ }^{70}$.

化反应。

\subsection{1甲醇光催化偶联制乙二醇}

乙二醇 $(E G)$ 作为聚对苯二甲酸乙二酯等重要 聚合物的关键原料, 在能源、化工等领域有着广泛 用途。当前乙二醇主要经乙烯环氧化和环氧乙烷 水合反应制备, 是高耗能、低效率过程。煤基合成 气经草酸二甲酯制乙二醇也是重要方法, 但工艺 流程长, 成本高。甲醇来源广泛, 可以由煤、天然 气或页岩气、生物质等碳资源乃至 $\mathrm{CO}_{2}$ 还原得到。 由甲醇出发制备乙二醇有望成为一条全新的原子 经济性高的非石油路线。

王野课题组 ${ }^{20}$ 发现 $\mathrm{CdS}$ 半导体具有独特的优 先活化甲醇 $\mathrm{C}-\mathrm{H}$ 键的能力, 故而具有很高的乙二 醇选择性。电子自旋共振(ESR)结果表明, 此反应 为自由基反应。若 $\mathrm{C}-\mathrm{H}$ 键被活化, 则产生 $\cdot \mathrm{CH}_{2} \mathrm{OH}$, 最终得到乙二醇; 若 $\mathrm{O}-\mathrm{H}$ 键被活化, 则产生 $\mathrm{CH}_{3} \mathrm{O}$, 最终得到甲酫。为了进一步研究 $\mathrm{X}-\mathrm{H}(\mathrm{X}=$ $\mathrm{C}$ 或O)活化模式, 该课题组研究了 $\mathrm{TiO}_{2}$ 与 $\mathrm{CdS}$ 两种 催化剂, 认为在 $\mathrm{TiO}_{2}$ 表面甲醇活化经历质子转移- 


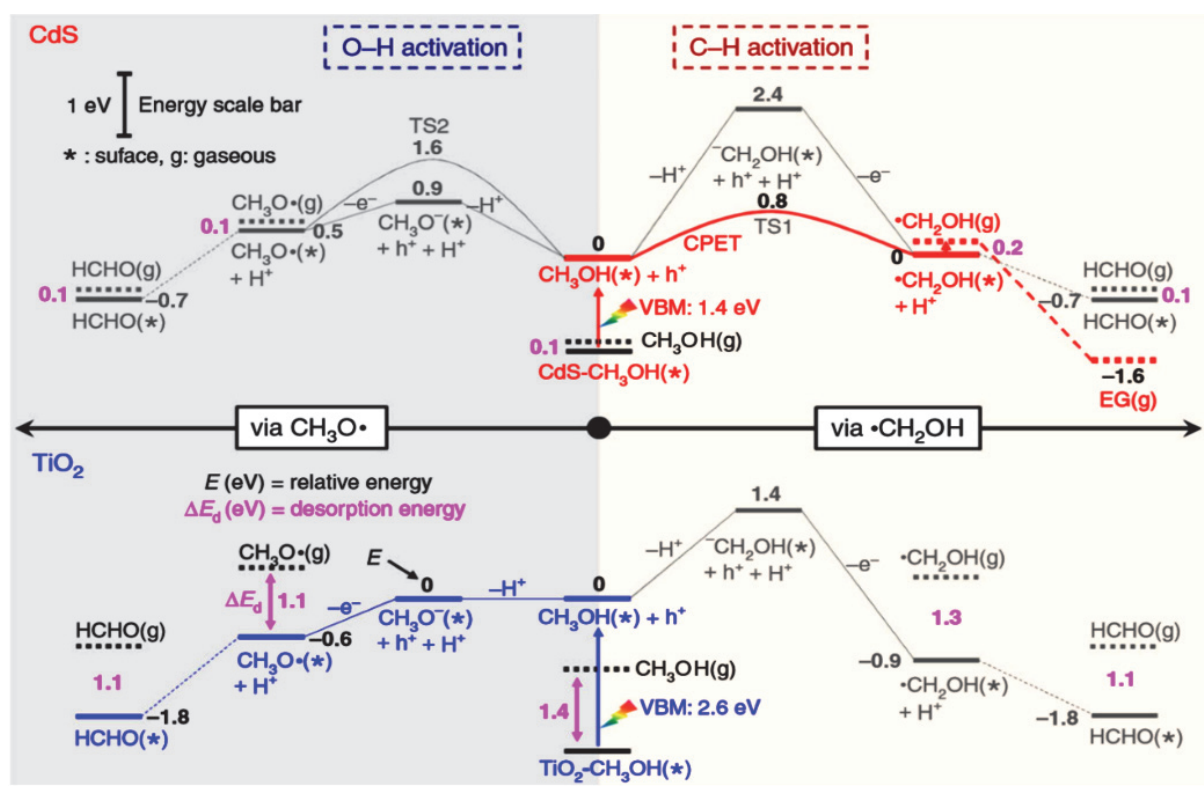

图8甲醇在 $\mathrm{CdS}(100)$ 和金红石 $\mathrm{TiO}_{2}(110)$ 面上 $\mathrm{C}-\mathrm{H}$ 和 $\mathrm{O}-\mathrm{H}$ 键活化能垒图

Fig. 8 Reaction energy profiles via $\mathrm{C}-\mathrm{H}$ and $\mathrm{O}-\mathrm{H}$ bond activation on $\mathrm{CdS}(100)$ and rutile $\mathrm{TiO}_{2}(110)$.

Reproduced with permission from reference ${ }^{20}$.

电子转移(PT-ET)两个过程, 在 $\mathrm{CdS}$ 上则经历质子电子协同转移(CPET)过程。DFT研究表明(图8), 在 $\mathrm{TiO}_{2}$ 表面上甲醇的吸附能为 $1.4 \mathrm{eV}$, 这表明甲醇与 二氧化钛表面相互作用力强, 故而易经由 PT得到 $\mathrm{CH}_{3} \mathrm{O}^{-}$, 再经ET得到 $\mathrm{CH}_{3} \mathrm{O} \cdot$ 。强吸附的 $\mathrm{CH}_{3} \mathrm{O} \cdot(1.1$ $\mathrm{eV}$ )很难从 $\mathrm{TiO}_{2}$ 表面脱附, 继续与空穴反应生成甲 醛。在 $\mathrm{CdS}$ 表面, 甲醇的吸附能只有 $-0.1 \mathrm{eV}$, 甲醇 与 $\mathrm{CdS}$ 作用力弱, 很难去质子化得到 $\mathrm{CH}_{3} \mathrm{O}^{-}$。此时, 甲醇活化经由CPET过程, $\mathrm{C}-\mathrm{H}$ 活化产生 $\cdot \mathrm{CH}_{2} \mathrm{OH}$ 的能垒 $(0.8 \mathrm{eV})$ 远低于 $\mathrm{O}-\mathrm{H}$ 活化产生 $\mathrm{CH}_{3} \mathrm{O}$ 的能 垒 $(1.6 \mathrm{eV})$, 故在 $\mathrm{CdS}$ 表面易产生 $\mathrm{CH}_{2} \mathrm{OH}$ 。弱吸附 的 $\cdot \mathrm{CH}_{2} \mathrm{OH}(0.2 \mathrm{eV})$ 易从 $\mathrm{CdS}$ 表面脱附, 偶联生成乙 二醇。此外, 他们采用具有优异电催化析氢性能的 $\mathrm{Mo}_{2} \mathrm{~S}$ 修饰 $\mathrm{CdS}$, 使乙二醇选择性提高到 $90 \%$, 生成 速率提高 20 倍。为了避免乙二醇过度氧化, 设计了 具有实时分离乙二醇功能的反应器, 最终乙二醇 选择性长时间保持在 $90 \%$, 最终收率可达 $16 \%$ 。

此外, 该课题组 ${ }^{85}$ 还发现光照下 $\mathrm{BiVO}_{4}$ 可以催 化活化甲醛并以 $\mathrm{C}-\mathrm{C}$ 偶联的方式生成 $\mathrm{C}_{2}$ 化合物 (乙二醇, 羟基乙醛等)。研究表明 $\mathrm{HCHO}$ 被光生空 穴活化产生 $\cdot \mathrm{CH}_{2} \mathrm{OH}$, 后通过自身偶联形成乙二 醇。而 $\mathrm{OH}^{-}$活化后产生· $\mathrm{OH}$, 进一步与 $\mathrm{HCHO}$ 反应 生成· $\mathrm{CHO}$, 再与 $\mathrm{CH}_{2} \mathrm{OH}$ 偶联则会产生羟基甲醛。 ESI数据表明 $\mathrm{V}^{4+}$ 浓度越高, $\mathrm{C}_{2}$ 选择性越好, 推测 $\mathrm{V}^{4+}$ 是催化 HCHO偶联的活性位点。通过进一步调 控 $\mathrm{BiVO}_{4}$ 的形貌 ${ }^{86}$, 乙二醇的产率可达 $11 \%, \mathrm{C}_{2}$ 产 物达 $21 \%$ 。
甲醇偶联制备乙二醇会产生氢气，而甲醛偶 联制备乙二醇需要两个质子, 若能直接将甲醇与 甲醛偶联制备乙二醇，将会是一条 $100 \%$ 原子经济 性的路径。我们课题组 ${ }^{72}$ 研究负载Pt的二氧化钛 (P25)在紫外光下催化甲醇与甲醛混合溶液制备乙 二醇的反应。研究结果如图9所示, 无光照或者无 催化剂时, 乙二醇产率几乎为零; 采用单独的甲醛 或者甲醇溶液, 仅有少量乙二醇产生; 当甲醇和甲 醛同时存在时, 乙二醇产率大大增加, 甲醇/甲醛 摩尔比为 $73 / 1$ 时乙二醇选择性可达 $80 \%$ 。EPR实验 及同位素标记实验表明, 单独甲醇光活化后产生 的. $\mathrm{CH}_{2} \mathrm{OH}$ 易与氢再次复合, 羟甲基利用率低, 而 加入 $\mathrm{HCHO}$ 后能快速捕获 $\cdot \mathrm{CH}_{2} \mathrm{OH}$, 从而提高羟甲 基的利用率及乙二醇选择性。

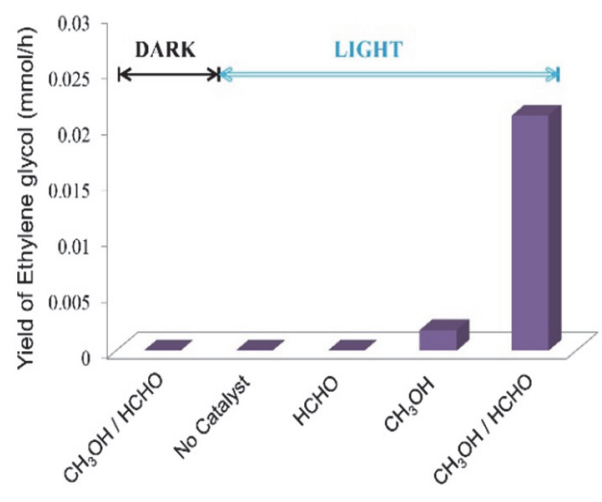

图9 乙二醇在不同反应条件下的收率

Fig. 9 Yield of EG under different reaction conditions.

Reproduced with permission from reference ${ }^{72}$. 


\subsection{2 甲醇光催化偶联制其它化学品}

高碳醇(碳数大于5)是表面活性剂、增塑剂的 原料。目前制备高碳醇通常是通过烯烃的氢甲酰 化反应得到醛, 再进行加氢反应得高碳醇。虽然氢 甲酰化工艺已经相对成熟, 但反应需要昂贵的铑 基催化剂。若能直接将烯烃与甲醇直接偶联制备 高碳醇, 无疑体现出很高的经济性 ${ }^{87}$ 。

我们课题组 ${ }^{73}$ 以 $\mathrm{Pt} / \mathrm{TiO}_{2}$ 为光催化剂, 在紫外光 照射下活化甲醇 $\mathrm{C}-\mathrm{H}$ 键并与高碳烯烃发生反马 氏加成, 可以得到高选择性的高碳伯醇。实验发现 纯 $\mathrm{TiO}_{2}$ 转化率较低, 但随着 $\mathrm{Pt}$ 负载量的增大, 单位 活性位转化的底物分子数(TON) 先增大后减少, 当 负载量为 $0.44 \%$ (质量分数) 时, $\mathrm{TON}$ 最高。这是因 为 $\mathrm{Pt}$ 能捕获 $\mathrm{TiO}_{2}$ 产生的光生电子, 阻止光生电子与 光生空穴复合, 进而提高TON, 但Pt过高则易发生 加氢反应得到饱和烷烃。同时, 我们还研究了甲醇 与不同碳链长度的烯烃的加成, 发现碳数越高, 反 马氏产物选择性越高, 选择性可从 $90 \%$ 提高到 $100 \%$ (表3)。EPR表明反应中间体为 $\cdot \mathrm{CH}_{2} \mathrm{OH}$, 被烯 烃端位碳捕获后, 再与质子反应则产生高选择性 的反马氏加成产物。此外, 在反应体系中加入适量 水能产生着基自由基, 活化甲醇生成稳定化合物,
提高高碳醇选择性。当醇底物扩展到乙醇、丙醇和 异丙醇时(表4), 仍然可发生 $\alpha$ 位 $\mathrm{C}-\mathrm{H}$ 断裂并与烯 烃进行反马氏加成, 且反应活性不断升高。

酮与甲醇的偶联反应, 一般为过渡金属催化 的甲基化反应 ${ }^{88,89}$, 或碘化钠催化的甲氧基化反 应 ${ }^{90}$ 。若采用光催化反应, 则能选择性活化 $\mathrm{C}-\mathrm{H}$ 发 生羟甲基化反应。

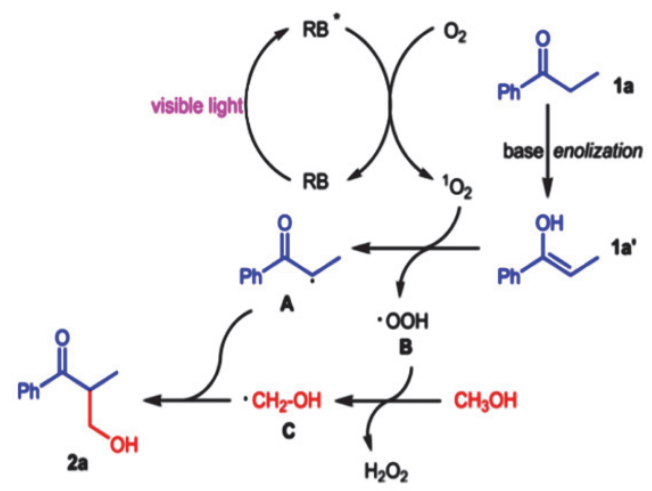

示意图8 酮的 $\alpha$-羟甲基化反应机理

Scheme 8 Proposed reaction mechanism for the $\alpha$-hydroxymethylation of ketones.

Reproduced with permission from reference ${ }^{91}$.

表3 甲醇与不同烯烃的偶联

Table 3 Hydrofunctionalization of olefins with methanol.

$$
H \otimes+R=\frac{\mathrm{P} U T \mathrm{TiO}_{2}}{\mathrm{Ar}_{1} \mathrm{UV}} \mathbb{R} \times
$$

$\mathrm{X}=-\mathrm{CH}_{2} \mathrm{OH} ;-\mathrm{CH}_{2} \mathrm{CN} ;-\mathrm{CH}_{2} \mathrm{COOH} ;-\mathrm{CH}_{2} \mathrm{COOC}_{2} \mathrm{H}_{5} ;-\mathrm{CH}_{2} \mathrm{COCH}_{3}$

\begin{tabular}{|c|c|c|c|c|c|c|c|}
\hline Entry & Substrate & Olefins & Main product & $10^{-3} \mathrm{TON}$ & $10^{-3} \mathrm{TOF} / \mathrm{h}^{-1}$ & Selectivity to target product $/ \%$ & Anti-Markovnikov regioselectivity $/ \%$ \\
\hline 1 & $-\mathrm{OH}$ & $\widehat{x+3}=$ & $\widehat{\mathrm{H}_{3}} \mathrm{\gamma OH}_{\mathrm{OH}}$ & 18.0 & 1.2 & 87.2 & 90 \\
\hline 2 & & 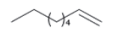 & & 33.1 & 2.2 & 84.0 & 92 \\
\hline 3 & & $\widehat{x r s}=$ & & 34.6 & 2.3 & 74.6 & 93 \\
\hline 4 & & $4 \%$ & $\mathrm{OH}$ & 45.3 & 3.0 & 62.3 & 93 \\
\hline 5 & & $\widehat{\gamma}+\frac{16}{8}$ & $\widehat{>} \times \mathrm{OH}_{\mathrm{OH}}$ & 14.9 & 1.0 & 54.4 & 100 \\
\hline 6 & & $\mathrm{HO}_{\mathrm{M}} \approx$ & $\mathrm{HO}_{\mathrm{N}} \mathrm{C}_{\mathrm{OH}}$ & 59.2 & 3.9 & 57.7 & 100 \\
\hline 7 & & & & 105.2 & 7.0 & 77.0 & 100 \\
\hline
\end{tabular}

TON: turnover number; TOF: turnover frequency. Reproduced with permission from reference ${ }^{73}$.

表4 不同醇底物与烯烃的偶联

Table 4 Investigation of the scope of alcohol substrates.

$\mathrm{R}_{1} \mathrm{CH}_{2} \mathrm{OH}+\mathrm{R}_{2}-\frac{\mathrm{PtTiO}_{2}}{\mathrm{Ar}, \mathrm{UV}} \rightarrow \mathrm{R}_{2} \mathrm{R}_{\mathrm{R}_{1}}^{\mathrm{OH}}$

\begin{tabular}{|c|c|c|c|c|c|c|c|}
\hline Entry & Substrate & Target product & $10^{-3} \mathrm{TON}$ & $10^{-3} \mathrm{TOF} / \mathrm{h}^{-1}$ & Selectivity to target product $\%$ & $\mathrm{~A} /(\mathrm{P}+\mathrm{A})$ & Anti-Markovnikov regioselectivity/\% \\
\hline 1 & $-\mathrm{OH}$ & $\mathrm{R}_{2} \sim \mathrm{OH}$ & 45.3 & 3.0 & 62.3 & 0.92 & 93 \\
\hline 2 & & $\mathrm{R}_{2}-$ & 83.2 & 5.6 & 86.8 & 0.97 & 100 \\
\hline 3 & & & 207.9 & 13.9 & 79.1 & 0.93 & 100 \\
\hline 4 & & & 282.6 & 28.3 & 79.7 & 0.86 & 100 \\
\hline
\end{tabular}

$\mathrm{A} /(\mathrm{P}+\mathrm{A}):$ alcohol/(paraffin + alcohol $)$ ratio. Reproduced with permission from reference ${ }^{73}$. 


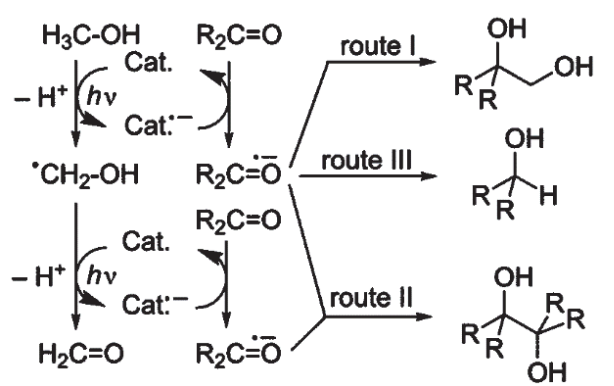

图10甲醇与羰基化合物光反应的三种反应途径

Fig. 10 Production forming routes I to III for photoredox catalysis of methanol/carbonyl compounds.

Reproduced with permission from reference ${ }^{92}$.

Yang 等 ${ }^{91}$ 探索了以甲醇为原料对甲氧基酮的 $\alpha-\mathrm{C}\left(s p^{3}\right) \mathrm{H}$ 进行羟甲基化的反应。以苯丙酮和甲醇 为底物, 虎红为光敏剂, 碳酸钾为碱, 室温可见光 下反应 $36 \mathrm{~h} ， \alpha$-羟甲基酮的产率最高可达 $53 \%$ 。该 反应为自由基历程, 如示意图8所示, 光敏剂受光 激发后将能量转移给氧气分子产生单线态 ${ }^{1} \mathrm{O}_{2}$, 酮 在碱的作用下转变为烯醇, 烯醇与单线态 ${ }^{1} \mathrm{O}_{2}$ 反应 产生酮的 $\alpha$ 碳自由基与 $\cdot \mathrm{OOH}$ 。 $\mathrm{OOH}$ 与甲醇反应生 成羟甲基自由基与酮的 $\alpha$ 碳自由基反应得到目标 产物。通过扩展酮底物, 发现无论是改变苯基上取 代基种类、增长碳链, 还是使用杂环芳香环, 反应 物产率都比较高, 且 $\alpha$ 碳的酸性越强, 产率越高。当 $\alpha$ 碳是叔碳时, 由于位阻效应过大, 产率仅有 $19 \%$, 与反应机理一致。

Griesbeck等 92 研究了紫外光下甲醇与酮的羰 基碳偶联反应, 并提出三种途径(图10): 一是甲醇 $\mathrm{C}-\mathrm{H}$ 键被光生空穴氧化得到羟甲基自由基, 而酮 得到电子被还原, 而后两者结合发生羟甲基化反 应; 二是酮活化还原后与自身反应得到邻二醇; 三 是酮与甲醇释放的 $\mathrm{H}$ 结合产生仲醇。以钛盐作为催 化剂时, 反应倾向于路线 I; 以异相催化剂和染料 为催化剂时, 倾向于路线 II; 但当采用 $\mathrm{TiO}_{2}$ 异相催 化剂时, 倾向于路线III, 其原因可能是 $\mathrm{TiO}_{2}$ 粒径较 大, 羟甲基受到扩散阻碍, 难以与酮的还原产物结 合，故易发生加氢还原反应。

\section{4 结论与展望}

甲烷既是燃料又是重要的化工原料, 其亲和 力差、与催化剂相互作用弱、 $\mathrm{C}-\mathrm{H}$ 键能高等特点 使得其难以活化, 甲烷催化转化一直以来都是化 学家们心中的“圣杯”。热催化甲烷转化条件苛刻, 且选择性较差。光催化反应由于其具有反应条件 温和、可操作性强、能耗低、副产物少等特点, 越 来越多地被用于甲烷转化。甲烷光催化转化主要
包括重整、氧化、偶联和基于LMCT跃迁反应。光 催化甲烷重整在于提高甲烷的极化和解离能力, 以 $\mathrm{Au} 、 \mathrm{Ni}$ 等金属在光照下具有等离子体共振效应为 代表, 通过光诱导产生高能电场, 增强对甲烷的极 化能力, 从而促进甲烷的活化。甲烷氧化制含氧产 物时, 需要避免甲烷过度氧化生成 $\mathrm{CO}_{2}$ 。光催化能 够在较温和的条件下实现甲烷的选择性转化, 减 少 $\mathrm{CO}_{2}$ 的生成。采用具有光催化活性的催化剂用于 含氧产物的制备, 通过调控催化剂的酸碱性质可 以得到高选择性的含氧产物, 对光催化剂进行修 饰, 提高催化剂表面羟基含量可以提高含氧产物 的生成速率, 而构建异质结(界面)也能够提高对高 能电子的利用率, 以上这些策略为制备高选择性 的含氧产物提供了思路。甲烷偶联反应要实现甲 烷的高转化率和高选择性可以从晶面的可控设 计、载流子的充分利用和载体孔道的篎选来实现。 在LMCT反应过程中, 主要构建高能烷氧自由基, 通过甲烷与烷氧自由基之间发生氢交换来实现甲 烷活化, 进一步转变为其它衍生物。

在甲醇催化转化中, 由于 $\mathrm{O}-\mathrm{H}$ 键会优先被活 化为甲氧基物种, 如何实现选择性 $\mathrm{C}-\mathrm{H}$ 活化是一 大挑战, 而光催化反应为选择性活化 $\mathrm{C}-\mathrm{H}$ 键得到 羟甲基提供了有效的途径。甲醇光催化转化反应 主要包括重整、氧化和羟甲基化偶联反应。甲醇重 整制氢反应机理为光激发半导体产生光生电子与 空穴, 光生空穴不断氧化甲醇, 逐步脱氢经由甲 醛、甲酸中间体得到二氧化碳与氢气。因此, 提高 光生空穴的氧化能力和光生载流子的分离效率尤 为重要。常在半导体上负载贵金属以捕获半导体 表面产生的光生电子, 避免光生电子与空穴的复 合, 提高光生载流子利用率。甲醇氧化反应则需要 调节控制催化剂的氧化能力, 避免过度氧化。除挑 选合适的氧化能力的半导体以外, 调控催化剂形 貌、尺寸以及表面处理也能改善其氧化效果。此 外, 通过修饰产生能带弯曲或者等离子效应可以 使一些本身无光催化活性的物质产生活性, 这对 促进光催化反应的发展有着重要意义。羟甲基化 反应仅需要活化 $\mathrm{C}-\mathrm{H}$ 键。因此, 设计选择性活化 甲醇中 $\mathrm{C}-\mathrm{H}$ 键的催化剂具有极大挑战性。此外, 为 了提高羟甲基自由基的利用效率, 常采用含双键 的不饱和物为反应底物以迅速捕获羟甲基自由 基, 或修饰催化剂表面结构促进羟甲基自身偶联。

尽管光催化在甲烷和甲醇活化中已经有较多 的研究, 如何更有效地利用光和提高光生载流子 分离及利用效率仍然是重要研究方向。围绕甲烷/ 甲醇高效光催化转化, 以下几个方面值得进一步 
研究: (1)设计具有特定晶面的光催化剂, 提升甲 烷/甲醇在择优晶面的解离活化能力; (2)调控光催 化剂的纳米结构, 促进光生载流子分离并抑制电 子和空穴复合; (3)合成高效中间体(自由基)捕获 剂, 从平衡角度推动甲烷/甲醇活化; (4)调节光催 化剂的氧化还原能力和酸碱性, 调控产物的氧化 还原程度，避免副产物生成。

\section{References}

(1) Herrerias, C. I.; Yao, X.; Li, Z.; Li, C. -J. Chem. Rev. 2007, 107, 2546. doi: $10.1021 / \mathrm{cr} 050980 \mathrm{~b}$

(2) Singh, M. K.; Akula, H. K.; Satishkumar, S.; Stahl, L.; Lakshman, M. K. ACS Catal. 2016, 6, 1921. doi: 10.1021/acscatal.5b02603

(3) Yu, J. -T.; Pan, C. Chem. Commun. 2016, 52, 2220. doi: $10.1039 / \mathrm{c} 5 \mathrm{cc} 08872 \mathrm{k}$

(4) Cook, A. K.; Schimler, S. D.; Matzger, A. J.; Sanford, M. S. Science 2016, 351, 1421. doi: 10.1126/science.aad9289

(5) Smith, K. T.; Berritt, S.; Gonzalez-Moreiras, M.; Ahn, S.; Smith, M. R., III; Baik, M. -H.; Mindiola, D. J. Science 2016, 351, 1424. doi: $10.1126 /$ science.aad 9730

(6) Takamatsu, K.; Hirano, K.; Satoh, T.; Miura, M. J. Org. Chem. 2015, 80, 3242. doi: 10.1021/acs.joc.5b00307

(7) Warratz, S.; Burns, D. J.; Zhu, C.; Korvorapun, K.; Rogge, T.; Scholz, J.; Jooss, C.; Gelman, D.; Ackermann, L. Angew. Chem. Int. Ed. 2017, 56, 1557. doi: 10.1002/anie.201609014

(8) Zhang, F. -L.; Hong, K.; Li, T. -J.; Park, H.; Yu, J. -Q. Science 2016, 351, 252. doi: $10.1126 /$ science.aad 7893

(9) Liao, K.; Negretti, S.; Musaev, D. G.; Bacsa, J.; Davies, H. M. L. Nature 2016, 533, 230. doi: 10.1038/nature17651

(10) Liao, K. B.; Pickel, T. C.; Oyarskikh, V. B.; Acsa, J. B.; Usaev, D. G M.; Davies, H. M. L. Nature 2017, 551, 609. doi: $10.1038 /$ nature 24641

(11) Liao, K.; Yang, Y.-F.; Li, Y.; Sanders, J. N.; Houk, K. N.; Musaev, D. G.; Davies, H. M. L. Nat. Chem. 2018, 551, 609. doi: $10.1038 / \mathrm{s} 41557-018-0087-7$

(12) Tzirakis, M. D.; Lykakis, I. N.; Orfanopoulos, M. Chem. Soc. Rev. 2009, 38, 2609. doi: 10.1039/B812100C

(13) Zhang, X.; MacMillan, D. W. C. J. Am. Chem. Soc. 2017, 139, 11353. doi: $10.1021 /$ jacs. $7 \mathrm{~b} 07078$

(14) Capacci, A. G.; Malinowski, J. T.; McAlpine, N. J.; Kuhne, J.; MacMillan, D. W. C. Nat. Chem. 2017, 9, 1073. doi: $10.1038 /$ nchem. 2797

(15) Maeda, H.; Takayama, H.; Segi, M. Photochem. Photobiol. Sci 2018, 17, 1118. doi: 10.1039/c8pp00239h

(16) Jeffrey, J. L.; Terrett, J. A.; MacMillan, D. W. C. Science 2015, 349, 1532. doi: $10.1126 /$ science.aac 8555
(17) Nicewicz, D. A.; MacMillan, D. W. C. Science 2008, 322, 77. doi: $10.1126 /$ science. 1161976

(18) Shaw, M. H.; Shurtleff, V. W.; Terrett, J. A.; Cuthbertson, J. D.; MacMillan, D. W. C. Science 2016, 352, 1304 doi: $10.1126 /$ science.aaf6635

(19) Zuo, Z.; Ahneman, D. T.; Chu, L.; Terrett, J. A.; Doyle, A. G.; MacMillan, D. W. C. Science 2014, 345, 437. doi: $10.1126 /$ science. 1255525

(20) Xie, S.; Shen, Z.; Deng, J.; Guo, P.; Zhang, Q.; Zhang, H.; Ma, C.; Jiang, Z.; Cheng, J.; Deng, D.; et al. Nat.Commun. 2018, 9, 1181. doi: 10.1038/s41467-018-03543-y

(21) Villa, K.; Murcia-López, S.; Morante, J. R.; Andreu, T. Appl. Catal. B 2016, 187, 30. doi: 10.1016/j.apcatb.2016.01.032

(22) Nomikos, G. N.; Panagiotopoulou, P.; Kondarides, D. I.; Verykios, X. E. Appl. Catal. B 2014, 146, 249. doi: 10.1016/j.apcatb.2013.03.018

(23) Chen, F.; Yang, Q.; Li, X.; Zeng, G.; Wang, D.; Niu, C.; Zhao, J.; An, H.; Xie, T.; Deng, Y. Appl. Catal. B 2017, 200, 330. doi: 10.1016/j.apcatb.2016.07.021

(24) Hammond, C.; Forde, M. M.; Ab Rahim, M. H.; Thetford, A.; He, Q.; Jenkins, R. L.; Dimitratos, N.; Lopez-Sanchez, J. A.; Dummer, N. F.; Murphy, D. M.; et al. Angew. Chem. Int. Ed. 2012, 51, 5129. doi: 10.1002/anie. 201108706

(25) Ab Rahim, M. H.; Forde, M. M.; Jenkins, R. L.; Hammond, C.; He, Q.; Dimitratos, N.; Lopez-Sanchez, J. A.; Carley, A. F.; Taylor, S. H.; Willock, D. J.; et al. Angew. Chem. Int. Ed. 2013, 52, 1280. doi: 10.1002/anie.201207717

(26) Forde, M. M.; Armstrong, R. D.; Hammond, C.; He, Q.; Jenkins, R. L.; Kondrat, S. A.; Dimitratos, N.; Lopez-Sanchez, J. A.; Taylor, S. H.; Willock, D.; et al. J. Am. Chem. Soc. 2013, 135, 11087. doi: $10.1021 / \mathrm{ja} 403060 \mathrm{n}$

(27) Agarwal, N.; Freakley, S. J.; McVicker, R. U.; Althahban, S. M.; Dimitratos, N.; He, Q.; Morgan, D. J.; Jenkins, R. L.; Willock, D. J.; Taylor, S. H.; et al. Science 2017, 358, 223. doi: $10.1126 /$ science.aan 6515

(28) Liu, H.; Meng, X.; Dao, T. D.; Zhang, H.; Li, P.; Chang, K.; Wang, T.; Li, M.; Nagao, T.; Ye, J. Angew. Chem. Int. Ed. 2015, 54, 11545. doi: 10.1002/anie.201504933

(29) Han, B.; Wei, W.; Chang, L.; Cheng, P.; Hu, Y. H. ACS Catal. 2015, 6, 494. doi: 10.1021/acscatal.5b02653

(30) Liu, H.; Meng, X.; Dao, T. D.; Liu, L.; Li, P.; Zhao, G.; Nagao, T.; Yang, L.; Ye, J. J. Mater. Chem. A 2017, 5, 10567. doi: $10.1039 / \mathrm{c} 7 \mathrm{ta} 00704 \mathrm{c}$

(31) Pan, F.; Xiang, X.; Deng, W.; Zhao, H.; Feng, X.; Li, Y. ChemCatChem 2018, 10, 940. doi: 10.1002/cctc.201701565

(32) Liu, H.; Dao, T. D.; Liu, L.; Meng, X.; Nagao, T.; Ye, J. Appl. Catal. B 2017, 209, 183. doi: 10.1016/j.apcatb.2017.02.080 
(33) Murcia-López, S.; Bacariza, M. C.; Villa, K.; Lopes, J. M.; Henriques, C.; Morante, J. R.; Andreu, T. ACS Catal. 2017, 7, 2878. doi: $10.1021 /$ acscatal.6b03535

(34) Sastre, F.; Fornes, V.; Corma, A.; Garcia, H. Chemistry 2012, 18, 1820. doi: $10.1002 /$ chem. 201102273

(35) Hu, Y.; Anpo, M.; Wei, C. J. Photochem. Photobiol. A 2013, 264, 48 doi: 10.1016/j.jphotochem.2013.05.005

(36) Li, L.; Fan, S.; Mu, X.; Mi, Z.; Li, C. J. J. Am. Chem. Soc. 2014, 136, 7793. doi: 10.1021/ja5004119

(37) Meng, L.; Chen, Z.; Ma, Z.; He, S.; Hou, Y.; Li, H.-H.; Yuan, R.; Huang, X.-H.; Wang, X.; Wang, X.; et al. Energ. Environ. Sci. 2018, $11,294$. doi: $10.1039 / \mathrm{c} 7 \mathrm{ee} 02951 \mathrm{a}$

(38) Li, L.; Li, G. D.; Yan, C.; Mu, X. Y.; Pan, X. L.; Zou, X. X.; Wang, K. X.; Chen, J. S. Angew. Chem. Int. Ed. 2011, 50, 8299. doi: 10.1002/anie. 201102320

(39) Li, L.; Cai, Y. Y.; Li, G. D.; Mu, X. Y.; Wang, K. X.; Chen, J. S. Angew. Chem. Int. Ed. 2012, 51, 4702. doi: 10.1002/anie.201200045

(40) Li, Z.; Mo, L.; Kathiraser, Y.; Kawi, S. ACS Catal. 2014, 4, 1526. doi: $10.1021 / \mathrm{cs} 401027 \mathrm{p}$

(41) Zhao, Y.; Kang, Y. Q.; Li, H.; Li, H. X. Green Chem. 2018, 20, 2781. doi: $10.1039 / \mathrm{c} 8 \mathrm{gc} 00743 \mathrm{~h}$

(42) Margossian, T.; Larmier, K.; Kim, S. M.; Krumeich, F.; Fedorov, A.; Chen, P.; Muller, C. R.; Coperet, C. J. Am. Chem. Soc. 2017, 139, 6919. doi: $10.1021 /$ jacs. 7 b01625

(43) Zhang, Y. X.; Dragan, A.; Geddes, C. D. J. Phys.Chem. C 2009, 113 , 15811. doi: 10.1021/jp900958n

(44) Xiong, Z.; Chen, X.; Wang, X.; Peng, L.; Yan, D.; Lei, H.; Fu, Y.; Wu, J.; Li, Z.; An, X.; et al. Appl. Surf. Sci. 2013, 268, 524. doi: 10.1016/j.apsusc.2012.12.161

(45) Nagli, L.; Gaft, M.; Gornushkin, I.; Glaus, R. Opt. Commun. 2016, 378, 41. doi: 10.1016/j.optcom.2016.05.071

(46) Kondratenko, V. A.; Berger-Karin, C.; Kondratenko, E. V. ACS Catal. 2014, 4, 3136. doi: 10.1021/cs5002465

(47) Park, J. H.; Kwon, Y.-i.; Nam, G. D.; Joo, J. H. J. Mater. Chem. A 2018, 6, 14246. doi: 10.1039/c8ta03021a

(48) Zhu, S.; Lian, X.; Fan, T.; Chen, Z.; Dong, Y.; Weng, W.; Yi, X.; Fang, W. Nanoscale 2018, 10, 14031. doi: 10.1039/c8nr02588f

(49) Sushkevich, V. L.; Palagin, D.; Ranocchiari, M.; van Bokhoven, J. A. Science 2017, 356, 523. doi: 10.1126/science.aam9035

(50) Khan, N. A.; Kennedy, E. M.; Dlugogorski, B. Z.; Adesina, A. A.; Stockenhuber, M. Catal. Commun. 2014, 53, 42. doi: 10.1016/j.catcom.2014.04.012

(51) Aslam, M.; Ismail, I. M.; Chandrasekaran, S.; Hameed, A. J. Hazard. Mater. 2014, 276, 120. doi: 10.1016/j.jhazmat.2014.05.022

(52) Tanaka, A.; Hashimoto, K.; Kominami, H. J. Am. Chem. Soc. 2014, 136, 586. doi: 10.1021/ja410230u
(53) Hameed, A.; Ismail, I. M. I.; Aslam, M.; Gondal, M. A. Appl. Catal. A 2014, 470, 327. doi: 10.1016/j.apcata.2013.10.045

(54) Li, R.; Han, H.; Zhang, F.; Wang, D.; Li, C. Energy Environ. Sci. 2014, 7, 1369. doi: 10.1039/c3ee43304h

(55) Ohkubo, K.; Hirose, K. Angew. Chem. Int. Ed. 2018, 57, 2126. doi: 10.1002/anie.201710945

(56) Keller, G. E.; Bhasin, M. M. J. Catal. 1982, 73, 9. doi: 10.1016/0021-9517(82)90075-6

(57) Brady, C.; Murphy, B.; Xu, B. ACS Catal. 2017, 7, 3924. doi: 10.1021/acscatal.7b00879

(58) Liu, Y.; Li, D.; Wang, T.; Liu, Y.; Xu, T.; Zhang, Y. ACS Catal. 2016, 6, 5366. doi: 10.1021/acscatal.6b01362

(59) Okolie, C.; Lyu, Y.; Kovarik, L.; Stavitski, E.; Sievers, C. ChemCatChem 2018, 10, 2700. doi: 10.1002/cctc.201701892

(60) Guo, J. J.; Hu, A.; Chen, Y.; Sun, J.; Tang, H.; Zuo, Z. Angew. Chem. Int. Ed. 2016, 55, 15319. doi: 10.1002/anie.201609035

(61) Hu, A.; Guo, J. J.; Pan, H.; Tang, H.; Gao, Z.; Zuo, Z. J. Am. Chem. Soc. 2018, 140, 1612. doi: 10.1021/jacs.7b13131

(62) Hu, A.; Guo, J. -J.; Pan, H.; Zuo, Z. Science 2018, 361, 668. doi: $10.1126 /$ science.aat 9750

(63) Su, L. -W.; Li, X. -R.; Sun, Z. -Y. Energ. Policy 2013, 63, 130. doi: 10.1016/j.enpol.2013.08.031

(64) Chiarello, G. L.; Aguirre, M. H.; Selli, E. J. Catal. 2010, 273, 182. doi: $10.1016 /$ j.jcat.2010.05.012

(65) Liu, Z.; Yin, Z.; Cox, C.; Bosman, M.; Qian, X.; Li, N.; Zhao, H.; Du, Y.; Li, J.; Nocera, D. G. Sci. Adv. 2016, 2, e1501425. doi: $10.1126 /$ sciadv. 1501425

(66) Liu, Y.; Yang, S.; Yin, S.-N.; Feng, L.; Zang, Y.; Xue, H. Chem. Eng. J. 2018, 334, 2401. doi: 10.1016/j.cej.2017.12.016

(67) DePuccio, D. P.; Landry, C. C. Catal. Sci.Technol. 2016, 6, 7512. doi: 10.1039/c6cy01449f

(68) Liu, J.; Han, C.; Yang, X.; Gao, G.; Shi, Q.; Tong, M.; Liang, X.; Li, C. J. Catal. 2016, 333, 162. doi: 10.1016/j.jcat.2015.11.005

(69) Liang, X.; Yang, X.; Gao, G.; Li, C.; Li, Y.; Zhang, W.; Chen, X.; Zhang, Y.; Zhang, B.; Lei, Y.; et al. J. Catal. 2016, 339, 68. doi: $10.1016 /$ j.jcat.2016.03.033

(70) Yang, X.; Zhang, A.; Gao, G.; Han, D.; Han, C.; Wang, J.; Lu, H.; Liu, J.; Tong, M. Catal. Commun. 2014, 43, 192. doi: 10.1016/j.catcom.2013.10.010

(71) Han, C. H.; Yang, X. Z.; Gao, G. J.; Wang, J.; Lu, H. L.; Liu, J.; Tong, M.; Liang, X. Y. Green Chem. 2014, 16, 3603. doi: $10.1039 / \mathrm{c} 4 \mathrm{gc} 00367 \mathrm{e}$

(72) Fan, Y. H.; Bao, J. X.; Shi, L.; Li, S. G.; Lu, Y. W.; Liu, H. J.; Wang, H.; Zhong, L. S.; Sun, Y. H. Catal. Lett. 2018, 148, 2274. doi: 10.1007/s10562-018-2465-6

(73) Fan, Y.; Li, S.; Bao, J.; Shi, L.; Yang, Y.; Yu, F.; Gao, P.; Wang, H.; Zhong, L.; Sun, Y. Green Chem. 2018, 20, 3450. 
doi: $10.1039 / \mathrm{c} 8 \mathrm{gc} 00971 \mathrm{f}$

(74) Chiarello, G. L.; Ferri, D.; Selli, E. J. Catal. 2011, 280, 168. doi: 10.1016/j.jcat.2011.03.013

(75) Highfield, J. G.; Chen, M. H.; Nguyen, P. T.; Chen, Z. Energ. Environ. Sci. 2009, 2. doi: 10.1039/b907781m

(76) Naldoni, A.; D’Arienzo, M.; Altomare, M.; Marelli, M.; Scotti, R.; Morazzoni, F.; Selli, E.; Dal Santo, V. Appl. Catal. B 2013, 130-131, 239. doi: 10.1016/j.apcatb.2012.11.006

(77) Guo, Q.; Xu, C.; Ren, Z.; Yang, W.; Ma, Z.; Dai, D.; Fan, H.; Minton, T. K.; Yang, X. J. Am. Chem. Soc. 2012, 134, 13366. doi: $10.1021 / \mathrm{ja} 304049 \mathrm{x}$

(78) Shen, M.; Henderson, M. A. J. Phys.Chem. C 2012, 116, 18788. doi: $10.1021 / \mathrm{jp} 3046774$

(79) Zhang, J.; Peng, C.; Wang, H.; Hu, P. ACS Catal. 2017, 7, 2374. doi: $10.1021 /$ acscatal.6b03348

(80) Iwase, Y.; Kobayashi, T.; Inazu, K.; Miyaji, A.; Baba, T. Catal. Lett. 2007, 118, 146. doi: 10.1007/s10562-007-9171-0

(81) Tang, X. C.; Zeng, Z. W.; Jiang, L. H.; Chen, L. A.; Wang, Z. M.; Jia, D. A. Z. Acta Chim. Sin. 2010, 68, 2013.

(82) Jogunola, O.; Salmi, T.; Kangas, M.; Mikkola, J. P. Chem. Eng. J. 2012, 203, 469. doi: 10.1016/j.cej.2012.06.085

(83) Lu, Z.; Gao, D.; Yin, H.; Wang, A.; Liu, S. J. Ind. Eng. Chem 2015,
31, 301. doi: 10.1016/j.jiec.2015.07.002

(84) Phillips, K. R.; Jensen, S. C.; Baron, M.; Li, S. C.; Friend, C. M. J. Am. Chem. Soc. 2013, 135, 574. doi: 10.1021/ja3106797

(85) Shen, Z. B.; Xie, S. J.; Fan, W. Q.; Zhang, Q. H.; Xie, Z. K.; Yang, W. M.; Wang, Y. D.; Lin, J. C.; Wu, X. J.; Wan, H. L.; et al. Catal. Sci.Technol. 2016, 6, 6485. doi: 10.1039/c6cy01468b

(86) Xie, S. J.; Shen, Z. B.; Zhang, H. M.; Cheng, J.; Zhang, Q. H.; Wang, Y. Catal. Sci. Technol. 2017, 7, 923. doi: 10.1039/c6cy02510b

(87) Pospech, J.; Fleischer, I.; Franke, R.; Buchholz, S.; Beller, M. Angew Chem. Int. Ed. 2013, 52, 2852. doi: 10.1002/anie.201208330

(88) Chan, L. K. M.; Poole, D. L.; Shen, D.; Healy, M. P.; Donohoe, T. J. Angew. Chem. Int. Ed. 2014, 53, 761. doi: 10.1002/anie.201307950

(89) Liu, Z. H.; Yang, Z. Z.; Yu, X. X.; Zhang, H. Y.; Yu, B.; Zhao, Y. F.; Liu, Z. M. Org. Lett. 2017, 19, 5228. doi: 10.1021 /acs.orglett.7b02462

(90) Zhu, C. J.; Zhang, Y. F.; Zhao, H. Q.; Huang, S. J.; Zhang, M.; Su, W. P. Adv. Synth. Catal. 2015, 357, 331. doi: 10.1002/adsc.201500006

(91) Yang, J.; Xie, D.; Zhou, H.; Chen, S.; Duan, J.; Huo, C.; Li, Z. Adv. Synth. Catal. 2018, 360, 3471. doi: 10.1002/adsc.201800467

(92) Griesbeck, A. G.; Reckenthaler, M. Beilstein J. Org. Chem. 2014, 10, 1143. doi: $10.3762 /$ bjoc. 10.114 